\title{
LIE ALGEBRA EXTENSIONS AND HIGHER ORDER COCYCLES
}

\author{
KARL-HERMANN NEEB
}

Communicated by Martin Schlichenmaier

\begin{abstract}
In this note we present an abstract approach, based on Lie algebra cohomology, to the Lie algebra extensions associated to symplectic manifolds. We associate to any Lie algebra cocycle of degree at least two an abelian extension by some space $\mathfrak{a}$ and central extensions of subalgebras analogous to the Lie algebras of symplectic, respectively, hamiltonian vector fields. We even obtain a Poisson bracket on $\mathfrak{a}$ compatible with the hamiltonian Lie subalgebra. We then describe how this general approach provides a unified treatment of cocycles defined by closed differential forms on Lie algebras of vector fields on possibly infinitedimensional manifolds.
\end{abstract}

\section{Introduction}

If $(M, \omega)$ is a finite-dimensional symplectic manifold, then we assign to each smooth function $f: M \rightarrow \mathbb{R}$ its hamiltonian vector field $X_{f}$ determined uniquely by $i_{X_{f}} \omega=\mathrm{d} f$, and this leads to a central extension of Lie algebras

$$
\mathbf{0} \rightarrow H_{\mathrm{dR}}^{0}(M, \mathbb{R}) \rightarrow\left(C^{\infty}(M, \mathbb{R}),\{\cdot, \cdot\}\right) \rightarrow \mathfrak{h a m}(M, \omega) \rightarrow \mathbf{0}
$$

where $\operatorname{ham}(M, \omega)$ denotes the Lie algebra of hamiltonian vector fields on $M$ and the Lie bracket on $C^{\infty}(M, \mathbb{R})$ is given by the Poisson bracket $\{f, g\}:=$ $\omega\left(X_{f}, X_{g}\right)$. Since a symplectic vector field $X$ on $M$ is hamiltonian if and only if the closed one-form $i_{X} \omega$ is exact and each closed one-form can be written as $i_{X} \omega$ for a symplectic vector field $X$, the exact sequence (1) can be extended to a four term exact sequence

$$
\mathbf{0} \rightarrow H_{\mathrm{dR}}^{0}(M, \mathbb{R}) \rightarrow C^{\infty}(M, \mathbb{R}) \rightarrow \mathfrak{s p}(M, \omega) \rightarrow H_{\mathrm{dR}}^{1}(M, \mathbb{R}) \rightarrow \mathbf{0}
$$

The central extension (1) can also be embedded into an abelian extension

$$
\mathbf{0} \rightarrow C^{\infty}(M, \mathbb{R}) \rightarrow \widehat{\mathcal{V}}(M):=C^{\infty}(M, \mathbb{R}) \oplus_{\omega} \mathcal{V}(M) \rightarrow \mathcal{V}(M) \rightarrow \mathbf{0}
$$


which is the abelian extension of the Lie algebra $\mathcal{V}(M)$ of smooth vector fields on $M$ by the module $C^{\infty}(M, \mathbb{R})$ defined by $\omega$, viewed as a Lie algebra two-cocycle on $\mathcal{V}(M)$ with values in $C^{\infty}(M, \mathbb{R})$. Moreover, (2) can be embedded into the exact sequence

$$
\begin{aligned}
\mathbf{0} \rightarrow H_{\mathrm{dR}}^{0}(M, \mathbb{R}) \rightarrow C^{\infty}(M, \mathbb{R}) \rightarrow \mathcal{V}(M) & \rightarrow \Omega^{1}(M, \mathbb{R}) / \mathrm{d} C^{\infty}(M, \mathbb{R}) \rightarrow \mathbf{0} \\
X & \mapsto\left[i_{X} \omega\right] .
\end{aligned}
$$

The first main point of this article is that this situation is a very special incarnation of vastly more general exact sequences arising from any Lie algebra $(p+2)$ cocycle $\omega \in Z^{p+2}(\mathfrak{g}, V)$ with values in a $\mathfrak{g}$-module $V$. A key idea, carried out in Section 1 , is that $\omega$ defines a sequence $\left(T_{m}(\omega)\right)_{0 \leq m \leq p+2}$ of Lie algebra cocycles

$$
T_{m}(\omega) \in Z^{m}\left(\mathfrak{g}, \bar{C}^{p+2-m}(\mathfrak{g}, V)\right), \quad T_{m}(\omega)\left(x_{1}, \ldots, x_{m}\right):=\left[i_{x_{m}} \ldots i_{x_{1}} \omega\right]
$$

where $\bar{C}^{k}(\mathfrak{g}, V):=C^{k}(\mathfrak{g}, V) / \mathrm{d}_{\mathfrak{g}}\left(C^{k-1}(\mathfrak{g}, V)\right)$ is endowed with the natural $\mathfrak{g}$ module structure. In Section 2, we further associate to $\omega$ two subalgebras $\mathfrak{h a m}(\mathfrak{g}, \omega)$ and $\mathfrak{s p}(\mathfrak{g}, \omega)$ of $\mathfrak{g}$ and consider the abelian Lie algebra extension $\widehat{\mathfrak{g}}=$ $\bar{C}_{c}^{p}(\mathfrak{g}, V) \oplus \Omega \mathfrak{g}$, corresponding to the cocycle $\Omega:=T_{2}(\omega)$, the associated flux cocycle $f_{\omega}:=T_{1}(\omega)$ and $\widehat{f}_{\omega}([\eta], x):=i_{x} \omega-\mathrm{d}_{\mathfrak{g}} \eta$ on $\widehat{\mathfrak{g}}$. With $\widehat{\mathfrak{h a m}}(\mathfrak{g}, \omega):=\operatorname{ker} \widehat{f_{\omega}}$ we then obtain the following diagram with exact rows (Lie algebra extensions) and exact columns (Theorem 13)

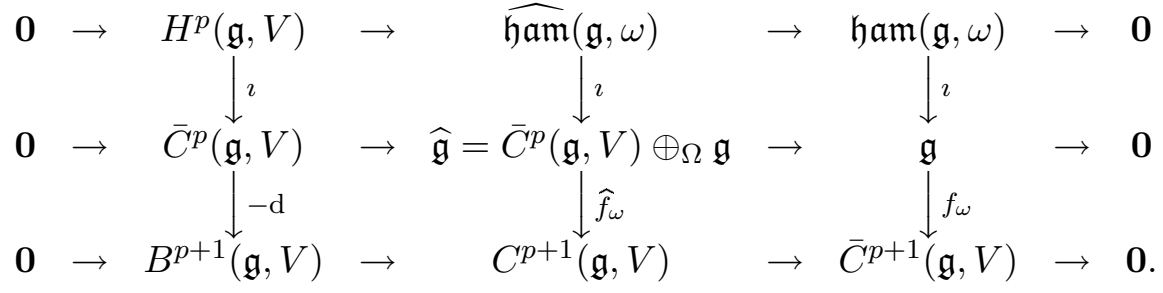

The inclusion of $\widehat{\mathfrak{h a m}}(\mathfrak{g}, \omega)$ into $\widehat{\mathfrak{g}}$ further defines a map $\widehat{\mathfrak{h a m}}(\mathfrak{g}, \omega) \rightarrow \bar{C}^{p}(\mathfrak{g}, V)$ whose kernel is an ideal, so that the its image inherits a Lie algebra structure, generalizing the Poisson bracket. We also have four term exact sequences enlarging the preceding ones

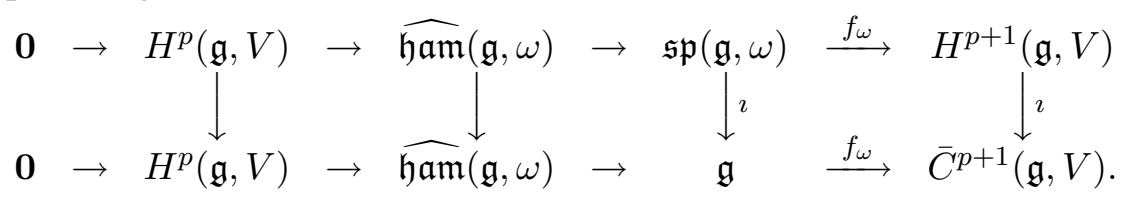

The corresponding classical situation in symplectic geometry is obtained as the special case with $\mathfrak{g}:=\mathcal{V}(M), p=0$ and $V:=C^{\infty}(M, \mathbb{R})$, which leads to $\mathfrak{s p}(\mathfrak{g}, \omega)=\mathfrak{s p}(M, \omega), \mathfrak{h} \mathfrak{a m}(\mathfrak{g}, \omega)=\mathfrak{h a m}(M, \omega)$ and $\widehat{\mathfrak{h a m}}(\mathfrak{g}, \omega) \cong C^{\infty}(M, \mathbb{R})$ 
endowed with the Poisson bracket. Here the exactness in the rightmost place in (2) follows from the non-degeneracy of the symplectic two-form $\omega$ on $M$, which is not required in the abstract context. This has the drawback that, not even for $p=0$, the flux cocycle $\mathfrak{s p}(\mathfrak{g}, \omega) \rightarrow H^{1}(\mathfrak{g}, V)$ is always surjective (Example 16).

In Section 3 we explain how the abstract Lie algebraic setup applies to $\mathfrak{g}=$ $\mathcal{V}(M)$, the Lie algebra of smooth vector fields on a smooth manifold $M$ and cocycles with values in $V:=C^{\infty}(M, E), E$ a locally convex space, represented by closed differential forms $\omega \in \Omega^{p+2}(M, E)$. In this context the natural replacement for the abstract space $\bar{C}_{c}^{p}(\mathfrak{g}, V)$ is the quotient space $\bar{\Omega}^{p}(M, E):=$ $\Omega^{p}(M, E) / \mathrm{d}\left(\Omega^{p-1}(M, E)\right)$. If $M$ is smoothly paracompact, then de Rham's Theorem holds (cf. [6]), which implies that the space of exact forms is closed, and thus $V$ inherits a natural (Hausdorff) locally convex topology turning it into a topological $\mathcal{V}(M)$-module whenever $M$ is finite-dimensional (otherwise the Lie bracket on $\mathcal{V}(M)$ is not continuous). Any closed $(p+2)$-form $\omega$ now leads to a twococycle $\Omega$ with values in $W:=\bar{\Omega}^{p}(M, E)$, and we obtain generalizations of the exact sequences (1) and (2). For the Lie algebra $\mathfrak{s p}(M, \omega)$ of $\omega$-symplectic vector fields we obtain a central extension by $W$ and show that if the singular homology group $H_{p}(M)$ is finitely generated, then it comes from an extension by the subspace $H_{\mathrm{dR}}^{p}(M, E)$. Integration over $p$-cycles now leads to interesting central extensions of $\mathfrak{s p}(M, \omega)$ only depending on the homology class of the cycle.

For $E=\mathbb{R}$ and $p=0$ the pair $(M, \omega)$ is a presymplectic manifold. In particular, we see that, up to the surjectivity of the flux cocycles, all the Lie algebraic structure contained in (1) and (2) is present without any non-degeneracy requirement on $\omega$ and without any restriction on $E$ or $p$.

Our setup also produces for a volume form $\mu$ on a compact $n$-dimensional manifold $M$ the universal central extension

$$
\mathbf{0} \rightarrow H_{\mathrm{dR}}^{n-2}(M, \mathbb{R}) \hookrightarrow \widehat{\mathfrak{h a m}}(M, \mu) \cong \bar{\Omega}^{n-2}(M, \mathbb{R}) \rightarrow \mathfrak{h a m}(M, \mu) \rightarrow \mathbf{0}
$$

due to Lichnerowicz ([7], [13]).

In Section 4 we consider a Lie algebra $\mathfrak{g}$ and a homomorphisms $\alpha: \mathfrak{g} \rightarrow \mathcal{V}(M)$, i.e., an infinitesimal action of $\mathfrak{g}$ on $M$. In this case we can obviously pull back all structure available on $\mathcal{V}(M)$, so that we derive in particular from each closed $E$ valued $(p+2)$-form an abelian extension of $\mathfrak{g}$ by $\bar{\Omega}^{p}(M, E)$. The new freedom we gain by this setup is that we may also assume that $E$ carries a $\mathfrak{g}$-module structure, which leads to a new action of $\mathfrak{g}$ on the spaces $\Omega^{p}(M, E)$ by $x . \omega:=\mathcal{L}_{\alpha(x)} \omega+$ $\rho_{E}(x) \circ \omega$, commuting with the exterior differential. The space of $\mathfrak{g}$-invariant forms on $M$ is a subcomplex of the de Rham complex which is of particular interest because evaluation in points $m \in M$ provide homomorphisms into the 
Lie algebra complex $\left(C_{c}(\mathfrak{g}, E), \mathrm{d}_{\mathfrak{g}}\right)$, hence geometric realizations of abstract Lie algebra cocycles.

If the action on $E$ is trivial, we obtain the notion of a generalized momentum map $\mu: \mathfrak{g} \rightarrow \bar{\Omega}^{p}(M, E)$ as a map whose image lies in the projection of $\widehat{\mathfrak{h a m}}(M, \omega)$ and which is a homomorphism with respect to the Poisson bracket.

We conclude with an appendix on the natural topology on $\mathcal{V}(M)$ and the space $\Omega^{p}(M, E)$ if $M$ is a locally convex manifold and $E$ a locally convex space.

We feel that the setup described in this note unifies special constructions of Lie algebra extensions one finds in the literature (mostly related to volume forms or symplectic forms) and that it also points into new directions such as the geometry of closed $p$-forms, which deserves a closer investigation. It also provides a natural bridge between Lie algebra cohomology and the geometry of differential forms from which both sides can profit. What we describe here is only the infinitesimal part of the picture. There is also a very interesting global side involving infinitedimensional Lie groups, their extensions, and their smooth actions on manifolds. The extra features of the global setup are the links to topology: the period homomorphisms and the flux cocycles ( [9], [10]). We plan to return to these global aspects in a forthcoming paper. Throughout this note we shall work in the context of topological Lie algebras and modules, which is needed in the global theory.

\section{The Transfer Map in Lie Algebra Cohomology}

In this section we introduce the transfer map in Lie algebra cohomology which is used to derive from a single Lie algebra $p$-cocycle a whole series of cocycles of degrees $\leq p$, but with values in more complicated modules. First we recall the basic facts from Lie algebra cohomology.

Definition 1. Let $\mathbb{K}$ be a topological field of characteristic zero. A topological Lie algebra $\mathfrak{g}$ is a $\mathbb{K}$-Lie algebra which is a topological vector space for which the Lie bracket is a continuous bilinear map. A topological $\mathfrak{g}$-module is a $\mathfrak{g}$-module $V$ which is a topological vector space for which the module structure, viewed as a map $\mathfrak{g} \times V \rightarrow V,(x, v) \mapsto x . v$, is continuous.

Throughout this section, $V$ denotes a topological module of the topological Lie algebra $\mathfrak{g}$. Note that this covers in particular the case where the topologies on $\mathbb{K}$, $\mathfrak{g}$ and $V$ are discrete, which simply leads to the algebraic setup where no topology is taken into account. 
Definition 2. For $p \in \mathbb{N}_{0}$, let $C_{c}^{p}(\mathfrak{g}, V)$ denote the space of continuous alternating maps $\mathfrak{g}^{p} \rightarrow V$, i.e., the Lie algebra $p$-cochains with values in the module $V$.

We identify $C_{c}^{0}(\mathfrak{g}, V)$ with $V$ and put $C_{c}(\mathfrak{g}, V):=\bigoplus_{p=0}^{\infty} C_{c}^{p}(\mathfrak{g}, V)$. We then obtain a cochain complex with the differentials $\mathrm{d}_{\mathfrak{g}}: C_{c}^{p}(\mathfrak{g}, V) \rightarrow C_{c}^{p+1}(\mathfrak{g}, V)$ given on $f \in C_{c}^{p}(\mathfrak{g}, V)$ by

$$
\begin{aligned}
\left(\mathrm{d}_{\mathfrak{g}} f\right)\left(x_{0}, \ldots, x_{p}\right):= & \sum_{j=0}^{p}(-1)^{j} x_{j} . f\left(x_{0}, \ldots, \widehat{x}_{j}, \ldots, x_{p}\right) \\
& +\sum_{i<j}(-1)^{i+j} f\left(\left[x_{i}, x_{j}\right], x_{0}, \ldots, \widehat{x}_{i}, \ldots, \widehat{x}_{j}, \ldots, x_{p}\right)
\end{aligned}
$$

where $\widehat{x}_{j}$ indicates omission of $x_{j}$ (see [2]). We call the operator $\mathrm{d}_{\mathfrak{g}}$ the Lie algebra differential. In view of $\mathrm{d}_{\mathfrak{g}}^{2}=0$, the space $Z_{c}^{p}(\mathfrak{g}, V):=\operatorname{ker}\left(\left.\mathrm{d}_{\mathfrak{g}}\right|_{C_{c}^{p}(\mathfrak{g}, V)}\right)$ of p-cocycles contains the space $B_{c}^{p}(\mathfrak{g}, V):=\mathrm{d}_{\mathfrak{g}}\left(C_{c}^{p-1}(\mathfrak{g}, V)\right)$ of $p$-coboundaries. The quotient

$$
H_{c}^{p}(\mathfrak{g}, V):=Z_{c}^{p}(\mathfrak{g}, V) / B_{c}^{p}(\mathfrak{g}, V)
$$

is the $p$-th continuous cohomology space of $\mathfrak{g}$ with values in the $\mathfrak{g}$-module $V$. We write $[f]:=f+B_{c}^{p}(\mathfrak{g}, V)$ for the cohomology class of the cocycle $f$.

If no topology is involved, i.e., $\mathbb{K}, \mathfrak{g}$ and $V$ are discrete, we omit the subscript $c$, hence write $C^{p}(\mathfrak{g}, V)$, instead of $C_{c}^{p}(\mathfrak{g}, V)$, etc.

Definition 3. On $C_{c}(\mathfrak{g}, V)$ we have a natural representation of $\mathfrak{g}$, given for $x \in \mathfrak{g}$ and $f \in C_{c}^{p}(\mathfrak{g}, V)$ by the Lie derivative

$$
\left(\mathcal{L}_{x} f\right)\left(x_{1}, \ldots, x_{p}\right)=x . f\left(x_{1}, \ldots, x_{p}\right)-\sum_{j=1}^{p} f\left(x_{1}, \ldots,\left[x, x_{j}\right], \ldots, x_{p}\right) .
$$

We further have for each $x \in \mathfrak{g}$ an insertion map $i_{x}: C_{c}(\mathfrak{g}, V) \rightarrow C_{c}(\mathfrak{g}, V)$ defined by $i_{x} f=0$ for $f \in C_{c}^{0}(\mathfrak{g}, V)=V$ and for $f \in C_{c}^{p}(\mathfrak{g}, V)$ by

$$
i_{x} f \in C_{c}^{p-1}(\mathfrak{g}, V), \quad\left(i_{x} f\right)\left(x_{1}, \ldots, x_{p-1}\right):=f\left(x, x_{1}, \ldots, x_{p-1}\right) .
$$

Remark 4. For $x, y \in \mathfrak{g}$ we have on $C_{c}(\mathfrak{g}, V)$ the Cartan formulas

$$
\mathcal{L}_{x}=\mathrm{d} \circ i_{x}+i_{x} \circ \mathrm{d}, \quad\left[\mathcal{L}_{x}, i_{y}\right]=i_{[x, y]}, \quad\left[\mathcal{L}_{x}, \mathrm{~d}_{\mathfrak{g}}\right]=0 .
$$

In particular, $\mathcal{L}_{x}\left(Z_{c}^{p}(\mathfrak{g}, V)\right) \subseteq B_{c}^{p}(\mathfrak{g}, V)$, so that the action of $\mathfrak{g}$ induced on $H_{c}^{p}(\mathfrak{g}, V)$ is trivial. We also obtain a natural $\mathfrak{g}$-action on the reduced cochain spaces

$$
\bar{C}_{c}^{p}(\mathfrak{g}, V):=C_{c}^{p}(\mathfrak{g}, V) / B_{c}^{p}(\mathfrak{g}, V) \supseteq H_{c}^{p}(\mathfrak{g}, V) .
$$


For $p, q \in \mathbb{N}_{0}$ we consider the injection

$$
\begin{aligned}
\widetilde{T}_{p} & : C_{c}^{p+q}(\mathfrak{g}, V) \rightarrow C^{p}\left(\mathfrak{g}, C_{c}^{q}(\mathfrak{g}, V)\right) \\
\left(\widetilde{T}_{p} f\right)\left(x_{1}, \ldots, x_{p}\right) & :=i_{x_{p}} \ldots i_{x_{1}} f .
\end{aligned}
$$

From the action of $\mathfrak{g}$ on the spaces $C_{c}^{q}(\mathfrak{g}, V)$ we obtain the Lie algebra differential

$$
\mathrm{d}_{\mathfrak{g}}^{\prime}: C^{p}\left(\mathfrak{g}, C_{c}^{q}(\mathfrak{g}, V)\right) \rightarrow C^{p+1}\left(\mathfrak{g}, C_{c}^{q}(\mathfrak{g}, V)\right)
$$

and we also have the linear maps

$$
\mathrm{d}_{\mathfrak{g}}^{\prime \prime}: C^{p}\left(\mathfrak{g}, C_{c}^{q}(\mathfrak{g}, V)\right) \rightarrow C^{p}\left(\mathfrak{g}, C_{c}^{q+1}(\mathfrak{g}, V)\right), \quad \omega \mapsto \mathrm{d}_{\mathfrak{g}} \circ \omega
$$

induced by the $\mathfrak{g}$-module homomorphisms $\mathrm{d}_{\mathfrak{g}}: C_{c}^{q}(\mathfrak{g}, V) \rightarrow C_{c}^{q+1}(\mathfrak{g}, V)$.

Lemma 5. For $p, q \in \mathbb{N}_{0}$ we have on $C_{c}^{p+q}(\mathfrak{g}, V)$ the identity

$$
\widetilde{T}_{p+1} \circ \mathrm{d}_{\mathfrak{g}}=\mathrm{d}_{\mathfrak{g}}^{\prime} \circ \widetilde{T}_{p}+(-1)^{p+1} \mathrm{~d}_{\mathfrak{g}}^{\prime \prime} \circ \widetilde{T}_{p+1} .
$$

Proof: (cf. Lemma 1 in [5]) We calculate for $x_{0}, \ldots, x_{p}, y_{1}, \ldots, y_{q} \in \mathfrak{g}$

$$
\begin{aligned}
& \left(\mathrm{d}_{\mathfrak{g}}^{\prime} \widetilde{T}_{p}(f)\right)\left(x_{0}, x_{1}, \ldots, x_{p}\right)\left(y_{1}, \ldots, y_{q}\right) \\
= & \sum_{j=0}^{p}(-1)^{j}\left(x_{j} . \widetilde{T}_{p} f\left(x_{0}, \ldots, \widehat{x}_{j}, \ldots, x_{p}\right)\right)\left(y_{1}, \ldots, y_{q}\right) \\
& +\sum_{0 \leq i<j \leq p}(-1)^{i+j}\left(\widetilde{T}_{p} f\right)\left(\left[x_{i}, x_{j}\right], x_{0}, \ldots, \widehat{x}_{i}, \ldots, \widehat{x}_{j}, \ldots, x_{p}\right)\left(y_{1}, \ldots, y_{q}\right) \\
= & \sum_{j=0}^{p}(-1)^{j}\left(x_{j} . f\left(x_{0}, \ldots, \widehat{x}_{j}, \ldots, x_{p}, y_{1}, \ldots, y_{q}\right)\right. \\
& \left.-\sum_{k=1}^{q} f\left(x_{0}, \ldots, \widehat{x}_{j}, \ldots, x_{p}, y_{1}, \ldots,\left[x_{j}, y_{k}\right], \ldots, y_{q}\right)\right) \\
& +\sum_{0 \leq i<j \leq p}(-1)^{i+j} f\left(\left[x_{i}, x_{j}\right], x_{0}, \ldots, \widehat{x}_{i}, \ldots, \widehat{x}_{j}, \ldots, x_{p}, y_{1}, \ldots, y_{q}\right) \\
= & \sum_{j=0}^{p}(-1)^{j} x_{j} . f\left(x_{0}, \ldots, \widehat{x}_{j}, \ldots, x_{p}, y_{1}, \ldots, y_{q}\right) \\
& -\sum_{j=0}^{p} \sum_{k=1}^{q}(-1)^{j+p+k-1} f\left(\left[x_{j}, y_{k}\right], x_{0}, \ldots, \widehat{x}_{j}, \ldots, x_{p}, y_{1}, \ldots, \widehat{y}_{k}, \ldots, y_{q}\right) \\
& +\sum_{0 \leq i<j \leq p}(-1)^{i+j} f\left(\left[x_{i}, x_{j}\right], x_{0}, \ldots, \widehat{x}_{i}, \ldots, \widehat{x}_{j}, \ldots, x_{p}, y_{1}, \ldots, y_{q}\right)
\end{aligned}
$$




$$
\begin{aligned}
& =\sum_{j=0}^{p}(-1)^{j} x_{j} \cdot f\left(x_{0}, \ldots, \widehat{x}_{j}, \ldots, x_{p}, y_{1}, \ldots, y_{q}\right) \\
& +\sum_{j=0}^{p} \sum_{k=1}^{q}(-1)^{j+(p+k)} f\left(\left[x_{j}, y_{k}\right], x_{0}, \ldots, \widehat{x}_{j}, \ldots, x_{p}, y_{1}, \ldots, \widehat{y}_{k}, \ldots, y_{q}\right) \\
& +\sum_{0 \leq i<j \leq p}(-1)^{i+j} f\left(\left[x_{i}, x_{j}\right], x_{0}, \ldots, \widehat{x}_{i}, \ldots, \widehat{x}_{j}, \ldots, x_{p}, y_{1}, \ldots, y_{q}\right) \\
& =\left(\mathrm{d}_{\mathfrak{g}} f\right)\left(x_{0}, \ldots, x_{p}, y_{1}, \ldots, y_{q}\right) \\
& -\sum_{k=1}^{q}(-1)^{p+k} y_{k} . f\left(x_{0}, \ldots, x_{p}, y_{1}, \ldots, \widehat{y}_{k}, \ldots, y_{q}\right) \\
& -\sum_{1 \leq j<k \leq q}(-1)^{2(p+1)+i+j} f\left(\left[y_{i}, y_{j}\right], x_{0}, \ldots, x_{p}, y_{1}, \ldots, \widehat{y}_{j}, \ldots, \widehat{y}_{k}, \ldots, y_{q}\right) \\
& =\left(\mathrm{d}_{\mathfrak{g}} f\right)\left(x_{0}, \ldots, x_{p}, y_{1}, \ldots, y_{q}\right) \\
& +(-1)^{p}\left[\sum_{k=1}^{q}(-1)^{k+1} y_{k} \cdot\left(\widetilde{T}_{p+1} f\left(x_{0}, \ldots, x_{p}\right)\left(y_{1}, \ldots, \widehat{y}_{k}, \ldots, y_{q}\right)\right)\right. \\
& \left.+\sum_{1 \leq j<k \leq q}(-1)^{i+j}\left(\widetilde{T}_{p+1} f\right)\left(x_{0}, \ldots, x_{p}\right)\left(\left[y_{i}, y_{j}\right], y_{1}, \ldots, \widehat{y}_{j}, \ldots, \widehat{y}_{k}, \ldots, y_{q}\right)\right] \\
& =\left(\widetilde{T}_{p+1}\left(\mathrm{~d}_{\mathfrak{g}} f\right)\left(x_{0}, \ldots, x_{p}\right)+(-1)^{p}\left(\mathrm{~d}_{\mathfrak{g}}^{\prime \prime} \widetilde{T}_{p+1} f\left(x_{0}, \ldots, x_{p}\right)\right)\right)\left(y_{1}, \ldots, y_{q}\right) \text {. }
\end{aligned}
$$

This proves the lemma.

Proposition 6. a) Composing $\widetilde{T}_{p}$ with the quotient map $C_{c}^{q}(\mathfrak{g}, V) \rightarrow \bar{C}_{c}^{q}(\mathfrak{g}, V)$, we obtain the $\mathfrak{g}$-equivariant transfer maps

$$
T_{p}: C_{c}^{p+q}(\mathfrak{g}, V) \rightarrow C^{p}\left(\mathfrak{g}, \bar{C}_{c}^{q}(\mathfrak{g}, V)\right)
$$

satisfying $T_{p+1} \circ \mathrm{d}_{\mathfrak{g}}=\mathrm{d}_{\mathfrak{g}}^{\prime} \circ T_{p}$, where $\mathrm{d}_{\mathfrak{g}}^{\prime}$ denotes the differential on the cochain complex $C\left(\mathfrak{g}, \bar{C}_{c}^{q}(\mathfrak{g}, V)\right)$. In particular, they induce linear maps

$$
T_{p}: H_{c}^{p+q}(\mathfrak{g}, V) \rightarrow H^{p}\left(\mathfrak{g}, \bar{C}_{c}^{q}(\mathfrak{g}, V)\right) .
$$

b) For each $\omega \in Z_{c}^{p+q}(\mathfrak{g}, V)$, the cocycle $\mathrm{d}_{\mathfrak{g}} \circ T_{p}(\omega) \in Z^{p}\left(\mathfrak{g}, C_{c}^{q+1}(\mathfrak{g}, V)\right)$ is a coboundary equal to $(-1)^{p-1} \mathrm{~d}_{\mathfrak{g}}^{\prime}\left(\widetilde{T}_{p-1}(\omega)\right)$.

Proof: a) In view of Lemma 5, it remains to verify the $\mathfrak{g}$-equivariance of $\widetilde{T}_{p}$. 
For $x, x_{1}, \ldots, x_{p}, y_{1}, \ldots, y_{q} \in \mathfrak{g}$ we have

$$
\begin{aligned}
& \left(\mathcal{L}_{x} \widetilde{T}_{p} f\right)\left(x_{1}, \ldots, x_{p}\right)\left(y_{1}, \ldots, y_{q}\right)=\left(\mathcal{L}_{x}\left(\widetilde{T}_{p} f\left(x_{1}, \ldots, x_{p}\right)\right)\right)\left(y_{1}, \ldots, y_{q}\right) \\
& -\sum_{i=1}^{p} \widetilde{T}_{p} f\left(x_{1}, \ldots,\left[x, x_{i}\right], \ldots, x_{p}\right)\left(y_{1}, \ldots, y_{q}\right) \\
= & x \cdot\left(\widetilde{T}_{p} f\left(x_{1}, \ldots, x_{p}\right)\left(y_{1}, \ldots, y_{q}\right)\right) \\
& -\sum_{k=1}^{q} \widetilde{T}_{p} f\left(x_{1}, \ldots, x_{p}\right)\left(y_{1}, \ldots,\left[x, y_{k}\right], \ldots, y_{q}\right) \\
& -\sum_{i=1}^{p} f\left(x_{1}, \ldots,\left[x, x_{i}\right], \ldots, x_{p}, y_{1}, \ldots, y_{q}\right) \\
= & x . f\left(x_{1}, \ldots, x_{p}, y_{1}, \ldots, y_{q}\right)-\sum_{k=1}^{q} f\left(x_{1}, \ldots, x_{p}, y_{1}, \ldots,\left[x, y_{k}\right], \ldots, y_{q}\right) \\
& -\sum_{i=1}^{p} f\left(x_{1}, \ldots,\left[x, x_{i}\right], \ldots, x_{p}, y_{1}, \ldots, y_{q}\right) \\
= & \left(\mathcal{L}_{x} f\right)\left(x_{1}, \ldots, x_{p}, y_{1}, \ldots, y_{q}\right)=\widetilde{T}_{p}\left(\mathcal{L}_{x} f\right)\left(x_{1}, \ldots, x_{p}\right)\left(y_{1}, \ldots, y_{q}\right) .
\end{aligned}
$$

b) is a consequence of Lemma 5 .

Definition 7. For each $\omega \in Z_{c}^{q}(\mathfrak{g}, V)$, the preceding lemma implies that we obtain a sequence $\left(T^{j}(\omega)\right)_{0 \leq j \leq q}$ of Lie algebra $j$-cocycles in $Z^{j}\left(\mathfrak{g}, \bar{C}_{c}^{q-j}(\mathfrak{g}, V)\right)$. We call these the cocycles derived from $\omega$. Of particular interest is the first derived cocycle

$$
f_{\omega}: \mathfrak{g} \rightarrow \bar{C}_{c}^{q-1}(\mathfrak{g}, V), \quad x \mapsto\left[i_{x} \omega\right]
$$

called the flux cocycle (cf. [9], [12]).

\section{Abelian and Central Extensions Defined by Higher Order Cocycles}

In this section we associate several interesting structures to a continuous $(p+2)$ cocycle $\omega \in Z_{c}^{p+2}(\mathfrak{g}, V)$. In particular, we define Lie subalgebras $\mathfrak{h a m}(\mathfrak{g}, \omega) \unlhd$ $\mathfrak{s p}(\mathfrak{g}, \omega) \subseteq \mathfrak{g}$ which behave in many respects like the Lie algebra of Hamiltonian, respectively, symplectic vector fields on a symplectic manifold. We further obtain central extensions of $\mathfrak{h a m}(\mathfrak{g}, \omega)$ and $\mathfrak{s p}(\mathfrak{g}, \omega)$ leading to the diagrams described in the introduction. 
We start with a simple lemma that creates a bridge between central and abelian extensions defined by invariant cocycles.

Lemma 8. If $\omega$ is a $\mathfrak{g}$-invariant element of $C^{p}(\mathfrak{g}, V)$ and $\mathrm{d}_{\mathfrak{g}}^{0}$ denotes the Lie algebra differential with respect to the trivial action of $\mathfrak{g}$ on $V$, then $\mathrm{d}_{\mathfrak{g}} \omega=-\mathrm{d}_{\mathfrak{g}}^{0} \omega$.

Proof: First we note that for $x_{0}, x_{1}, \ldots, x_{p} \in \mathfrak{g}$ the invariance of $\omega$ implies that

$$
\begin{aligned}
& x_{i} . \omega\left(x_{0}, \ldots, \widehat{x}_{i}, \ldots, x_{p}\right) \\
&=\omega\left(\left[x_{i}, x_{0}\right], x_{1}, \ldots, \widehat{x}_{i}, \ldots, x_{p}\right)+\ldots+\omega\left(x_{0}, \ldots, \widehat{x}_{i}, \ldots,\left[x_{i}, x_{p}\right]\right) \\
&=\sum_{j=0}^{i-1}(-1)^{j} \omega\left(\left[x_{i}, x_{j}\right], \ldots, \widehat{x}_{j}, \ldots, \widehat{x}_{i}, \ldots, x_{p}\right) \\
&+\sum_{j=i+1}^{p}(-1)^{j+1} \omega\left(\left[x_{i}, x_{j}\right], \ldots, \widehat{x}_{i}, \ldots, \widehat{x}_{j}, \ldots, x_{p}\right) .
\end{aligned}
$$

This leads to

$$
\begin{aligned}
& \sum_{i=0}^{p}(-1)^{i} x_{i} . \omega\left(x_{0}, \ldots, \widehat{x}_{i}, \ldots, x_{p}\right) \\
&=\sum_{j<i}(-1)^{j+i} \omega\left(\left[x_{i}, x_{j}\right], \ldots, \widehat{x}_{j}, \ldots, \widehat{x}_{i}, \ldots, x_{p}\right) \\
&+\sum_{j>i}(-1)^{i+j+1} \omega\left(\left[x_{i}, x_{j}\right], \ldots, \widehat{x}_{i}, \ldots, \widehat{x}_{j}, \ldots, x_{p}\right) \\
&= \sum_{j<i}(-1)^{j+i} \omega\left(\left[x_{i}, x_{j}\right], \ldots, \widehat{x}_{j}, \ldots, \widehat{x}_{i}, \ldots, x_{p}\right) \\
&+\sum_{j<i}(-1)^{i+j+1} \omega\left(\left[x_{j}, x_{i}\right], \ldots, \widehat{x}_{j}, \ldots, \widehat{x}_{i}, \ldots, x_{p}\right) \\
&= \sum_{j<i}(-1)^{j+i} \omega\left(\left[x_{i}, x_{j}\right], \ldots, \widehat{x}_{j}, \ldots, \widehat{x}_{i}, \ldots, x_{p}\right) \\
&+\sum_{j<i}(-1)^{i+j} \omega\left(\left[x_{i}, x_{j}\right], \ldots, \widehat{x}_{j}, \ldots, \widehat{x}_{i}, \ldots, x_{p}\right) \\
&= 2 \sum_{j<i}(-1)^{i+j} \omega\left(\left[x_{i}, x_{j}\right], \ldots, \widehat{x}_{j}, \ldots, \widehat{x}_{i}, \ldots, x_{p}\right) \\
&=-2\left(\mathrm{~d}_{\mathfrak{g}}^{0} \omega\right)\left(x_{0}, \ldots, x_{p}\right) .
\end{aligned}
$$

We conclude that $\mathrm{d}_{\mathfrak{g}} \omega=-2 \mathrm{~d}_{\mathfrak{g}}^{0} \omega+\mathrm{d}_{\mathfrak{g}}^{0} \omega=-\mathrm{d}_{\mathfrak{g}}^{0} \omega$. 
Proposition 9. For any cocycle $\omega \in Z_{c}^{q}(\mathfrak{g}, V)$, we define the Lie subalgebras of symplectic, respectively, hamiltonian elements of $\mathfrak{g}$ $\mathfrak{s p}(\mathfrak{g}, \omega):=\left\{x \in \mathfrak{g} ; \mathcal{L}_{x} \omega=0\right\}, \quad \mathfrak{h a m}(\mathfrak{g}, \omega):=\left\{x \in \mathfrak{g} ; i_{x} \omega \in B_{c}^{q-1}(\mathfrak{g}, V)\right\}$.

Then the following assertions hold:

1) The restriction of $\omega$ to $\mathfrak{s p}(\mathfrak{g}, \omega)$ is a cocycle with respect to the trivial module structure on $V$.

2) $[\mathfrak{s p}(\mathfrak{g}, \omega), \mathfrak{s p}(\mathfrak{g}, \omega)] \leq \mathfrak{h a m}(\mathfrak{g}, \omega) \leq \mathfrak{s p}(\mathfrak{g}, \omega)$. In particular, $\mathfrak{h a m}(\mathfrak{g}, \omega)$ is an ideal of $\mathfrak{s p}(\mathfrak{g}, \omega)$.

3) For $p>0$ the flux cocycle $f_{\omega}: \mathfrak{g} \rightarrow \bar{C}_{c}^{q-1}(\mathfrak{g}, V), x \mapsto\left[i_{x} \omega\right]$ satisfies

$$
\mathfrak{h a m}(\mathfrak{g}, \omega)=\operatorname{ker} f_{\omega} \quad \text { and } \quad \mathfrak{s p}(\mathfrak{g}, \omega)=f_{\omega}^{-1}\left(H_{c}^{q-1}(\mathfrak{g}, V)\right) .
$$

\section{Proof:}

1) follows from Lemma 8.

2) For $x, y \in \mathfrak{s p}(\mathfrak{g}, \omega)$ we have $0=\mathcal{L}_{x} \omega=\mathrm{d}_{\mathfrak{g}}\left(i_{x} \omega\right)$, showing that $\mathfrak{h a m}(\mathfrak{g}, \omega) \subseteq$ $\mathfrak{s p}(\mathfrak{g}, \omega)$. We further get with Remark 4

$$
i_{[x, y]} \omega=\left[\mathcal{L}_{x}, i_{y}\right] \omega=\mathcal{L}_{x} i_{y} \omega=\mathrm{d}_{\mathfrak{g}}\left(i_{x} i_{y} \omega\right)=\mathrm{d}_{\mathfrak{g}}\left(T_{2}(\omega)(y, x)\right) .
$$

3) The first relation is a reformulation of the definition of $\mathfrak{h a m}(\mathfrak{g}, \omega)$, and the second one follows from $\mathrm{d}_{\mathfrak{g}}\left(i_{x} \omega\right)=\mathcal{L}_{x} \omega$ for $x \in \mathfrak{g}$ (Remark 4).

Remark 10. a) For $q=0$ and $\omega \in Z_{c}^{q}(\mathfrak{g}, V)=V^{\mathfrak{g}}$ we have $\mathfrak{s p}(\mathfrak{g}, \omega)=\mathfrak{g}=$ $\mathfrak{h a m}(\mathfrak{g}, \omega)$.

b) For $q=1$ and $\omega \in Z_{c}^{1}(\mathfrak{g}, V)$ we have

$$
\mathfrak{s p}(\mathfrak{g}, \omega)=\omega^{-1}\left(V^{\mathfrak{g}}\right) \quad \text { and } \quad \mathfrak{h a m}(\mathfrak{g}, \omega)=\operatorname{ker} \omega .
$$

Comparing b) in the preceding remark with statement 3 of Proposition 9, it is natural to compare $H_{c}^{q}(\mathfrak{g}, V)$ with the set of $\mathfrak{g}$-fixed points in $\bar{C}_{c}^{q}(\mathfrak{g}, V)$. The following lemma provides a sufficient condition for equality which is satisfied in many applications.

Lemma 11. If $B_{c}^{p+1}(\mathfrak{g}, V)^{\mathfrak{g}}=\{0\}$, then $\bar{C}_{c}^{p}(\mathfrak{g}, V)^{\mathfrak{g}}=H_{c}^{p}(\mathfrak{g}, V)$.

Proof: We consider the exact sequence of $\mathfrak{g}$-modules

$$
\mathbf{0} \rightarrow H_{c}^{p}(\mathfrak{g}, V) \hookrightarrow \bar{C}_{c}^{p}(\mathfrak{g}, V) \stackrel{\overline{\mathrm{d}}_{\mathfrak{g}}}{\longrightarrow} B_{c}^{p+1}(\mathfrak{g}, V) \rightarrow \mathbf{0}
$$

where $\overline{\mathrm{d}}_{\mathfrak{g}}[\eta]:=\mathrm{d}_{\mathfrak{g}} \eta$. In view of the $\mathfrak{g}$-equivariance of $\mathrm{d}_{\mathfrak{g}}$ (Remark 4$), \overline{\mathrm{d}}_{\mathfrak{g}}$ maps $\bar{C}_{c}^{p}(\mathfrak{g}, V)^{\mathfrak{g}}$ into $B_{c}^{p+1}(\mathfrak{g}, V)^{\mathfrak{g}}=\{0\}$. Therefore $\bar{C}_{c}^{p}(\mathfrak{g}, V)^{\mathfrak{g}} \subseteq \operatorname{ker}\left(\overline{\mathrm{d}}_{\mathfrak{g}}\right)=H_{c}^{p}(\mathfrak{g}, V)$, and since $\mathfrak{g}$ acts trivially on $H_{c}^{p}(\mathfrak{g}, V)$ (Definition 2), the assertion follows. 
Now let $\omega \in Z_{c}^{p+2}(\mathfrak{g}, V)$. Then $\Omega:=T_{2}(\omega) \in Z^{2}\left(\mathfrak{g}, \bar{C}_{c}^{p}(\mathfrak{g}, V)\right)$ is a two-cocycle (Definition 7), defining an abelian Lie algebra extension $\widehat{\mathfrak{g}}:=\bar{C}_{c}^{p}(\mathfrak{g}, V) \oplus_{\Omega} \mathfrak{g}, \quad\left[([\eta], x),\left(\left[\eta^{\prime}\right], x^{\prime}\right)\right]=\left(x \cdot\left[\eta^{\prime}\right]-x^{\prime} \cdot[\eta]+\Omega\left(x, x^{\prime}\right),\left[x, x^{\prime}\right]\right)$ of $\mathfrak{g}$ by $\bar{C}_{c}^{p}(\mathfrak{g}, V)$ (cf. [10], Rem. II.9, for more details on the classification of topologically split abelian extensions by two-cocycles).

Lemma 12. a) The restriction $\Omega_{0}$ of $\Omega$ to $\mathfrak{s p}(\mathfrak{g}, \omega)$ is a Lie algebra cocycle with values in $\bar{C}_{c}^{p}(\mathfrak{g}, V)$, considered as a trivial $\mathfrak{s p}(\mathfrak{g}, \omega)$-module and the composition $\mathrm{d}_{\mathfrak{g}} \circ \Omega_{0} \in Z^{2}\left(\mathfrak{s p}(\mathfrak{g}, \omega), C_{c}^{p+1}(\mathfrak{g}, V)\right)$ is a coboundary.

b) In the central extension $\widehat{\mathfrak{s p}}(\mathfrak{g}, \omega):=\bar{C}_{c}^{p}(\mathfrak{g}, V) \oplus_{-\Omega_{0}} \mathfrak{s p}(\mathfrak{g}, \omega)$, the subspace

$$
\widehat{\mathfrak{h a m}}(\mathfrak{g}, \omega):=\left\{([\eta], x) \in \widehat{\mathfrak{s p}}(\mathfrak{g}, \omega) ; \mathrm{d}_{\mathfrak{g}} \eta=i_{x} \omega\right\}
$$

is an ideal containing all commutators. The projection map $q: \widehat{\mathfrak{h a m}}(\mathfrak{g}, \omega) \rightarrow$ $\mathfrak{h a m}(\mathfrak{g}, \omega),([\eta], x) \mapsto x$ defines a central extension

$$
\mathbf{0} \rightarrow H_{c}^{p}(\mathfrak{g}, V) \hookrightarrow \widehat{\mathfrak{h a m}}(\mathfrak{g}, \omega) \rightarrow \mathfrak{h a m}(\mathfrak{g}, \omega) \rightarrow \mathbf{0} .
$$

c) Let $\bar{C}_{c}^{p}(\mathfrak{g}, V)_{\omega}:=\left\{[\eta] \in \bar{C}_{c}^{p}(\mathfrak{g}, V)\right.$; for which there exists $x \in \mathfrak{g}$ such that $\left.\mathrm{d}_{\mathfrak{g}} \eta=i_{x} \omega\right\}$. Then the Poisson bracket

$$
\left\{[\eta],\left[\eta^{\prime}\right]\right\}:=\Omega\left(x^{\prime}, x\right) \quad \text { for } \quad \mathrm{d}_{\mathfrak{g}} \eta=i_{x} \omega, \quad \mathrm{d}_{\mathfrak{g}} \eta^{\prime}=i_{x^{\prime}} \omega
$$

defines on $\bar{C}_{c}^{p}(\mathfrak{g}, V)_{\omega}$ the structure of a Lie algebra whose center is the space $\bar{C}_{c}^{p}(\mathfrak{g}, V)^{\mathfrak{h a m}(\mathfrak{g}, \omega)}$, and the projection map

$$
p: \widehat{\mathfrak{h a m}}(\mathfrak{g}, \omega) \rightarrow \bar{C}_{c}^{p}(\mathfrak{g}, V)_{\omega}, \quad([\eta], x) \mapsto[\eta]
$$

is a surjective homomorphism of Lie algebras.

Proof: a) Since the transfer map $T_{p}$ is $\mathfrak{g}$-equivariant (see Proposition 6), $\Omega$ is $\mathfrak{s p}(\mathfrak{g}, \omega)$-invariant, so that its restriction $\Omega_{0}$ to $\mathfrak{s p}(\mathfrak{g}, \omega)$ is an invariant cocycle, hence also a cocycle with respect to the trivial action (Lemma 8). That $\mathrm{d}_{\mathfrak{g}} \circ \Omega_{0}$ is a coboundary follows from $\mathrm{d}_{\mathfrak{g}}(\Omega(x, y))=-i_{[x, y]} \omega=\left(\mathrm{d}_{\mathfrak{g}} f_{\omega}\right)(x, y)$ (cf. (6)).

b) From equation (6) it also follows that $\widehat{\mathfrak{h a m}}(\mathfrak{g}, \omega)$ contains all commutators of $\widehat{\mathfrak{s p}}(\mathfrak{g}, \omega)$. The kernel of the projection map $q: \widehat{\mathfrak{h a m}}(\mathfrak{g}, \omega) \rightarrow \mathfrak{h a m}(\mathfrak{g}, \omega)$ coincides with $H_{c}^{p}(\mathfrak{g}, V)$, so that we obtain a central extension of $\mathfrak{h a m}(\mathfrak{g}, \omega)$ by this space. 
c) The kernel of the projection map $p$ is $\mathfrak{n}:=\left\{(0, x) \in \widehat{\mathfrak{h a m}}(\mathfrak{g}, \omega) ; i_{x} \omega=0\right\}$. For any $([\eta], y) \in \widehat{\mathfrak{h a m}}(\mathfrak{g}, \omega)$ and $(0, x) \in \mathfrak{n}$ we have

$$
[(0, x),([\eta], y)]=(-\Omega(x, y),[x, y])=\left(-\left[i_{y} i_{x} \omega\right],[x, y]\right)=(0,[x, y])
$$

showing that $\mathfrak{n}$ is an ideal. Hence the quotient space $\bar{C}_{c}^{p}(\mathfrak{g}, V)_{\omega}$ carries a unique Lie bracket $\{\cdot, \cdot\}$ for which $p$ is a quotient homomorphism. It is given for $\mathrm{d}_{\mathfrak{g}} \eta=$ $i_{x} \omega$ and $\mathrm{d}_{\mathfrak{g}} \eta^{\prime}=i_{x^{\prime}} \omega$ by

$$
\left\{[\eta],\left[\eta^{\prime}\right]\right\}:=p\left(\left[([\eta], x),\left(\left[\eta^{\prime}\right], x^{\prime}\right)\right]\right)=\Omega\left(x^{\prime}, x\right) .
$$

To verify the statement on the center of $\bar{C}_{c}^{p}(\mathfrak{g}, V)_{\omega}$, we observe that $[\eta]$ with $\mathrm{d}_{\mathfrak{g}} \eta=$ $i_{x} \omega$ is central if and only if for each $x^{\prime} \in \mathfrak{h a m}(\mathfrak{g}, \omega)$ we have

$$
0=\Omega\left(x^{\prime}, x\right)=-\left[i_{x^{\prime}} i_{x} \omega\right]=-\left[i_{x^{\prime}} \mathrm{d}_{\mathfrak{g}} \eta\right]=-\left[\mathcal{L}_{x^{\prime}} \eta\right]=-x^{\prime} \cdot[\eta] .
$$

We collect the main points of this section in the following theorem.

Theorem 13. Let $\omega \in Z_{c}^{p+2}(\mathfrak{g}, V)$.

a) The inclusion map $\phi: \widehat{\mathfrak{h a m}}(\mathfrak{g}, \omega) \rightarrow \widehat{\mathfrak{g}}$ is a homomorphism of Lie algebras whose range is the kernel of the cocycle

$$
\widehat{f}_{\omega}: \widehat{\mathfrak{g}} \rightarrow C_{c}^{p+1}(\mathfrak{g}, V), \quad([\eta], x) \mapsto i_{x} \omega-\mathrm{d}_{\mathfrak{g}} \eta .
$$

We thus obtain the following commutative diagram of Lie algebra extensions (in the rows) with short exact columns

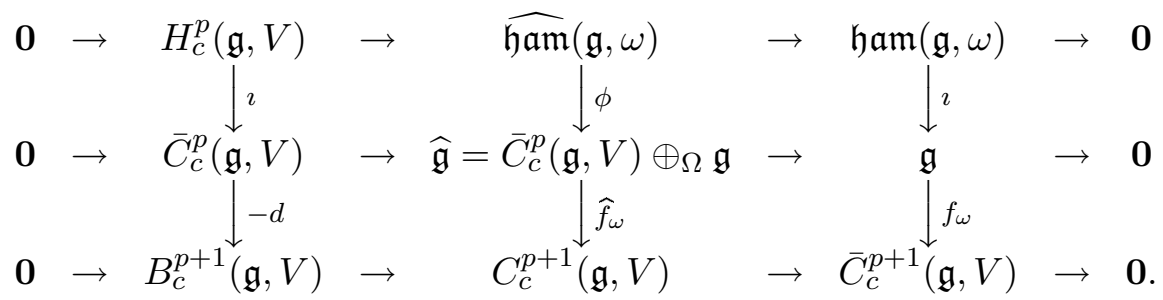

b) The following commutative diagram has exact rows

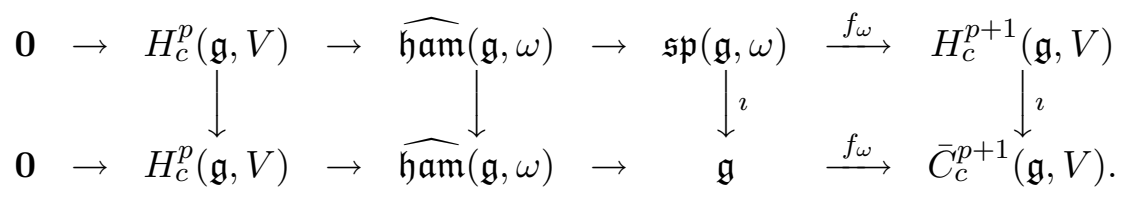


Proof: a) In view of Lemma 12, it remains to see that $\phi$ is a homomorphism of Lie algebras and that $\widehat{f}_{\omega}$ is a cocycle. The first assertion follows from the fact that the bracket of $([\eta], x),\left(\left[\eta^{\prime}\right], x^{\prime}\right) \in \widehat{\mathfrak{h a m}}(\mathfrak{g}, \omega)$ in $\widehat{\mathfrak{g}}$ is given by

$$
\begin{aligned}
{\left[([\eta], x),\left(\left[\eta^{\prime}\right], x^{\prime}\right)\right] } & =\left(\left[\mathcal{L}_{x} \eta^{\prime}-\mathcal{L}_{x^{\prime}} \eta\right]+\Omega\left(x, x^{\prime}\right),\left[x, x^{\prime}\right]\right) \\
& =\left(\left[i_{x} \mathrm{~d}_{\mathfrak{g}} \eta^{\prime}-i_{x^{\prime}} \mathrm{d}_{\mathfrak{g}} \eta\right]+\Omega\left(x, x^{\prime}\right),\left[x, x^{\prime}\right]\right) \\
& =\left(\left[i_{x} i_{x^{\prime}} \omega-i_{x^{\prime}} i_{x} \omega\right]+\Omega\left(x, x^{\prime}\right),\left[x, x^{\prime}\right]\right) \\
& =\left(2 \Omega\left(x^{\prime}, x\right)+\Omega\left(x, x^{\prime}\right),\left[x, x^{\prime}\right]\right)=\left(-\Omega\left(x, x^{\prime}\right),\left[x, x^{\prime}\right]\right) .
\end{aligned}
$$

To see that $\widehat{f}_{\omega}$ is a cocycle, we first observe that $\widehat{f}_{\omega}=q^{*}\left(\widetilde{T}_{1}(\omega)\right)-\mathrm{d}_{\mathfrak{g}} \circ \theta$, where $\theta \in C^{1}\left(\widehat{\mathfrak{g}}, \bar{C}_{c}^{p}(\mathfrak{g}, V)\right)$ is the projection onto $\bar{C}^{p}(\mathfrak{g}, V)$, i.e., $\theta([\eta], x)=[\eta]$. In view of $q^{*} \Omega=-d_{\widehat{\mathfrak{g}}} \theta$ in $Z^{2}\left(\widehat{\mathfrak{g}}, \bar{C}_{c}^{p}(\mathfrak{g}, V)\right)$ (cf. [10], Rem. VI.1), we get with Proposition 6

$$
\begin{aligned}
d_{\widehat{\mathfrak{g}}} \widehat{f}_{\omega} & =q^{*}\left(\mathrm{~d}_{\mathfrak{g}} \widetilde{T}_{1}(\omega)\right)-\mathrm{d}_{\mathfrak{g}} \circ\left(d_{\widehat{\mathfrak{g}}} \theta\right)=q^{*}\left(\mathrm{~d}_{\mathfrak{g}} \widetilde{T}_{1}(\omega)\right)+\mathrm{d}_{\mathfrak{g}} \circ\left(q^{*} \Omega\right) \\
& =q^{*}\left(\mathrm{~d}_{\mathfrak{g}} \widetilde{T}_{1}(\omega)+\mathrm{d}_{\mathfrak{g}} \circ T_{2}(\omega)\right)=0 .
\end{aligned}
$$

The remaining assertions are clear.

b) The exactness of the rows follows from Lemma 12 .

Remark 14. Let $\omega \in Z_{c}^{2}(\mathfrak{g}, V)$ and $\widehat{\mathfrak{g}}=V \oplus_{\omega} \mathfrak{g}$ the corresponding abelian extension of $\mathfrak{g}$ by $V$ with $q(v, x)=x$. For $(v, x) \in \widehat{\mathfrak{g}}$ we then have

$$
\operatorname{ad}(v, x)\left(v^{\prime}, x^{\prime}\right)=\left[(v, x),\left(v^{\prime}, x^{\prime}\right)\right]=\left(x \cdot v^{\prime}-x^{\prime} \cdot v+\omega\left(x, x^{\prime}\right),\left[x, x^{\prime}\right]\right)
$$

so that the linear map $\operatorname{ad}(v, x)$ preserves the subspace $\{0\} \times \mathfrak{g} \cong \mathfrak{g}$ of $\widehat{\mathfrak{g}}$ if and only if $i_{x} \omega=\mathrm{d}_{\mathfrak{g}} v$, which, in view of Theorem 13 , leads to

$$
\widehat{\mathfrak{h a m}}(\mathfrak{g}, \omega) \cong\{(v, x) \in \widehat{\mathfrak{g}} ; \operatorname{ad}(v, x)(\{0\} \times \mathfrak{g}) \subseteq\{0\} \times \mathfrak{g}\} .
$$

In particular, an element $x \in \mathfrak{g}$ lifts to an element of $\widehat{\mathfrak{g}}$ preserving $\{0\} \times \mathfrak{g}$ if and only if it is hamiltonian.

For the projection map $\theta: \widehat{\mathfrak{g}} \rightarrow V,(v, x) \mapsto v$ and $(v, x) \in \widehat{\mathfrak{g}}$, we further have

$$
((v, x) \cdot \theta)\left(v^{\prime}, x^{\prime}\right)=x \cdot v^{\prime}-\theta\left(\left[(v, x),\left(v^{\prime}, x^{\prime}\right)\right]\right)=x^{\prime} \cdot v-\omega\left(x, x^{\prime}\right)=-\widehat{f}_{\omega}(v, x)\left(x^{\prime}\right)
$$

so that the cocycle $\widehat{f}_{\omega}$ measures to which extent the adjoint action of an element of $\widehat{\mathfrak{g}}$ preserves the subspace $\operatorname{ker} \theta=\{0\} \times \mathfrak{g}$. 
Example 15. If $V$ is a trivial $\mathfrak{g}$-module and $p=2$, then $\mathcal{L}_{x} \omega=\mathrm{d}_{\mathfrak{g}}\left(i_{x} \omega\right)$ vanishes if and only if $i_{x} \omega$ vanishes on the commutator algebra $[\mathfrak{g}, \mathfrak{g}]$, hence $\mathfrak{s p}(\mathfrak{g}, \omega)=$ $[\mathfrak{g}, \mathfrak{g}]^{\perp \omega}$. Further $B_{c}^{1}(\mathfrak{g}, V)=\{0\}$ leads to $\mathfrak{h} \mathfrak{a m}(\mathfrak{g}, \omega)=\operatorname{rad}(\omega)$. For $V=\mathbb{R}, a$ necessary condition for

$$
f_{\omega}: \mathfrak{s p}(\mathfrak{g}, \omega) \rightarrow H_{c}^{1}(\mathfrak{g}, V)=\operatorname{Lin}(\mathfrak{g} /[\mathfrak{g}, \mathfrak{g}], V)
$$

to be surjective is that $\operatorname{rad}(\omega) \subseteq[\mathfrak{g}, \mathfrak{g}]$. If, conversely, $\mathfrak{g}$ is finite-dimensional and $\operatorname{rad}(\omega) \subseteq[\mathfrak{g}, \mathfrak{g}]$, then the fact that the induced form on $\mathfrak{g} / \operatorname{rad}(\omega)$ is non-degenerate implies that $f_{\omega}$ is surjective.

Example 16. If $\mathfrak{g}$ is the three-dimensional Heisenberg algebra and $\omega$ is a nonzero two-cocycle on $\mathfrak{g}$, then $\operatorname{rad}(\omega)$ is one-dimensional, and $\operatorname{rad}(\omega) \subseteq[\mathfrak{g}, \mathfrak{g}]=$ $\mathfrak{z}(\mathfrak{g})$ is equivalent to $\operatorname{rad}(\omega)=[\mathfrak{g}, \mathfrak{g}]$. This implies that $\omega$ is a coboundary. Therefore the flux homomorphism $f_{\omega}: \mathfrak{s p}(\mathfrak{g}, \omega) \rightarrow H^{1}(\mathfrak{g}, \mathbb{R})$ is surjective if and only of $\omega$ is a coboundary. If this is not the case, then $\mathfrak{s p}(\mathfrak{g}, \omega)$ is a proper twodimensional subalgebra containing the center and $\operatorname{rad}(\omega)$, and its image under $f_{\omega}$ is a one-dimensional subspace of the two-dimensional space $H^{1}(\mathfrak{g}, \mathbb{R})$.

Remark 17. From the embedding of $\widehat{\mathfrak{h a m}}(\mathfrak{g}, \omega)$ as an ideal into $\widehat{\mathfrak{s p}}(\mathfrak{g}, \omega)$, we obtain an action of the Lie algebra $\mathfrak{s p}(\mathfrak{g}, \omega)$ on $\widehat{\mathfrak{h a m}}(\mathfrak{g}, \omega)$ by

$$
x .\left(\left[\eta^{\prime}\right], x^{\prime}\right):=\left(-\Omega\left(x, x^{\prime}\right),\left[x, x^{\prime}\right]\right) .
$$

Since this action extends the canonical action of $\mathfrak{h a m}(\mathfrak{g}, \omega)$ on its central extension $\widehat{\mathfrak{h a m}}(\mathfrak{g}, \omega)$, and the homomorphism $q_{\sigma}: \widehat{\mathfrak{h a m}}(\mathfrak{g}, \omega) \rightarrow \mathfrak{s p}(\mathfrak{g}, \omega)$ is equivariant, it defines a so-called crossed module (cf. [10], [17]). To any crossed module one associates a characteristic class, which in our case is an element of

$$
H^{3}\left(\mathfrak{s p}(\mathfrak{g}, \omega) / \mathfrak{h a m}(\mathfrak{g}, \omega), H_{c}^{p}(\mathfrak{g}, V)\right) \cong C^{3}\left(\mathfrak{s p}(\mathfrak{g}, \omega) / \mathfrak{h} \mathfrak{a m}(\mathfrak{g}, \omega), H_{c}^{p}(\mathfrak{g}, V)\right)
$$

From [10] we know that the existence of the $\mathfrak{s p}(\mathfrak{g}, \omega)$-equivariant embedding of $\widehat{\mathfrak{h a m}}(\mathfrak{g}, \omega)$ into $\widehat{\mathfrak{s p}}(\mathfrak{g}, \omega)$ implies that the characteristic class of this crossed module vanishes.

\section{Applications to Lie Algebras of Vector Fields}

In this section $M$ denotes a smooth manifold modeled on a locally convex space, $\mathfrak{g}:=\mathcal{V}(M)$ the Lie algebra of smooth vector fields on $M$ (which is not topological if $\operatorname{dim} M=\infty$ ) and $E$ a Mackey complete locally convex space. Recall that 
Mackey completeness means that Riemann integrals of smooth curves $[0,1] \rightarrow E$ exist (cf. [6], Theorem 2.14). Then $V:=C^{\infty}(M, E)$ carries a natural topological $\mathfrak{g}$-module structure and so do the spaces $\Omega^{p}(M, E)$ and $\bar{\Omega}^{p}(M, E):=$ $\Omega^{p}(M, E) / d\left(\Omega^{p-1}(M, E)\right)$ (see the Appendix). The space of closed $E$-valued $p$-forms is denoted $Z_{\mathrm{dR}}^{p}(M, E)$ and the subspace of exact $E$-valued $p$-forms by $B_{\mathrm{dR}}^{p}(M, E)$, so that $H_{\mathrm{dR}}^{p}(M, E)=Z_{\mathrm{dR}}^{p}(M, E) / B_{\mathrm{dR}}^{p}(M, E)$ is a subspace of $\bar{\Omega}^{p}(M, E)$.

Remark 18. a) If each vector $v \in T_{p}(M)$ can be extended to a smooth vector field on $M$, then the natural morphism

$$
(\Omega(M, E), \mathrm{d}) \hookrightarrow\left(C_{c}(\mathfrak{g}, V), \mathrm{d}_{\mathfrak{g}}\right)
$$

of cochain complexes is injective. Moreover, we have $\mathrm{d}_{\mathfrak{g}} V=\mathrm{d} C^{\infty}(M, E) \subseteq$ $\Omega^{1}(M, E) \subseteq C_{c}^{1}(\mathfrak{g}, V)$, so that the natural map $\bar{\Omega}^{1}(M, E) \rightarrow \bar{C}_{c}^{1}(\mathfrak{g}, V)$ is injective.

b) The assumptions of a) are in particular satisfied if $M$ is diffeomorphic to an open subset of a locally convex space, hence they are always satisfied locally.

c) If $M$ is modeled on a smoothly paracompact locally convex space, then we can use smooth partitions of unity to extend vectors to vector fields, so that the assumption of a) is satisfied. If, in addition, $M$ is smoothly paracompact, then de Rham's Theorem holds (cf. [6], Theorem 34.7), and the subspace of exact forms in $\Omega^{p}(M, E)$ is the common kernel of the integration maps over smooth $p$ cocycles, which are continuous linear maps. Hence $B_{\mathrm{dR}}^{p}(M, E)$ is closed, which implies that $\bar{\Omega}^{p}(M, E)$ inherits a Hausdorff locally convex topology. If $M$ is finitedimensional, this turns it into a topological $\mathcal{V}(M)$-module.

Since we do not know for which locally convex manifold $M$ the natural maps

$$
\bar{\Omega}^{p}(M, E) \rightarrow \bar{C}^{p}\left(\mathcal{V}(M), C^{\infty}(M, E)\right)
$$

are injective for $p>1$, we have to use a slight modification of the transfer maps in the context of smooth manifolds, but this also leads to more geometric information

Definition 19. For any smooth manifold $M$ we define transfer maps

$$
\begin{aligned}
\widetilde{T}_{p}^{M}: & \Omega^{p+q}(M, E) \rightarrow C^{p}\left(\mathcal{V}(M), \Omega^{q}(M, E)\right) \\
\left(\widetilde{T}_{p}^{M} \omega\right)\left(X_{1}, \ldots, X_{p}\right) & :=i_{X_{p}} \ldots i_{X_{1}} \omega
\end{aligned}
$$

and, accordingly

$$
\begin{aligned}
T_{p}^{M} & : \Omega^{p+q}(M, E) \rightarrow C^{p}\left(\mathcal{V}(M), \bar{\Omega}^{q}(M, E)\right) \\
\left(T_{p}^{M} \omega\right)\left(X_{1}, \ldots, X_{p}\right) & :=\left[i_{X_{p}} \ldots i_{X_{1}} \omega\right] .
\end{aligned}
$$


Lemma 20. For $p, q \in \mathbb{N}_{0}$ we have on $\Omega^{p+q}(M, E)$ the identity

$$
\widetilde{T}_{p+1}^{M} \circ \mathrm{d}=\mathrm{d}_{\mathcal{V}(M)} \circ \widetilde{T}_{p}^{M}+(-1)^{p+1} \mathrm{~d} \circ T_{p+1}^{M} .
$$

Proof: If each vector $v \in T_{p}(M)$ extends to a smooth vector field on $M$, this follows immediately from Lemma 5 because $\Omega^{p}(M, E)$ embeds into the space $C\left(\mathcal{V}(M), C^{\infty}(M, E)\right)$. As this assumption is always satisfied locally (Remark 18), the assertion follows.

Definition 21. For $\omega \in Z_{\mathrm{dR}}^{q}(M, E)$ we define the Lie subalgebras of symplectic, respectively, hamiltonian vector fields on $M$ by

$$
\mathfrak{s p}(M, \omega):=\left\{X ; \mathcal{L}_{X} \omega=0\right\}, \quad \mathfrak{h a m}(M, \omega):=\left\{X ; i_{X} \omega \in B_{\mathrm{dR}}^{q-1}(M, E)\right\} .
$$

For $q>0$ the flux cocycle $f_{\omega}: \mathcal{V}(M) \rightarrow \bar{\Omega}^{q-1}(M, E), X \mapsto\left[i_{X} \omega\right]$ then satisfies

$$
\mathfrak{h a m}(M, \omega)=\operatorname{ker} f_{\omega} \quad \text { and } \quad \mathfrak{s p}(M, \omega)=f_{\omega}^{-1}\left(H_{\mathrm{dR}}^{q-1}(M, E)\right)
$$

(Proposition 9).

We now obtain for each closed $\omega \in \Omega^{p+2}(M, E)$ some two-cocycle $\Omega \in Z^{2}\left(\mathcal{V}(M), \bar{\Omega}^{p}(M, E)\right)$. If $M$ is finite-dimensional, this cocycle defines a topologically split abelian extension

$$
\widehat{\mathfrak{g}}=\bar{\Omega}^{p}(M, E) \oplus \Omega \mathcal{V}(M)
$$

Moreover, we obtain central extensions $\widehat{\mathfrak{s p}}(M, \omega)$ of $\mathfrak{s p}(M, \omega)$ by $\bar{\Omega}^{p}(M, E)$ and $\widehat{\mathfrak{h a m}}(M, \omega)$ of $\mathfrak{h a m}(M, \omega)$ by the space $H_{\mathrm{dR}}^{p}(M, E)$.

If $p=0$, then $\omega$ is a closed two-form, $\bar{\Omega}^{0}(M, E)=C^{\infty}(M, E)$, and $H_{\mathrm{dR}}^{0}(M, E)$ is the subspace of locally constant functions. On the subspace

$$
C^{\infty}(M, E)_{\omega}:=\left\{f \in C^{\infty}(M, E) ; \text { there exists } X \in \mathcal{V}(M) \mathrm{d} f=i_{X} \omega\right\}
$$

we further obtain a Lie algebra structure by the Poisson bracket

$$
\{f, g\}:=\Omega\left(X_{g}, X_{f}\right), \quad \text { for } \quad \mathrm{d} f=i_{X_{f}} \omega, \quad \mathrm{d} g=i_{X_{g}} \omega \text {. }
$$

If, in addition, $E=\mathbb{R}$, then the cokernel of $f_{\omega}$ very much depends on the regularity of $\omega$. For $\omega=0$ we have $\mathfrak{s p}(M, \omega)=\mathcal{V}(M), f_{\omega}=0$, and therefore $\operatorname{coker}\left(f_{\omega}\right)=H_{\mathrm{dR}}^{1}(M, E)$. The opposite situation arises if $\omega$ is strongly symplectic in the sense that it induces a bijection $\mathcal{V}(M) \rightarrow \Omega^{1}(M, \mathbb{R}), X \mapsto i_{X} \omega$ and coker $\left(f_{\omega}\right)=\{0\}$. In particular, we then have $C^{\infty}(M, \mathbb{R})_{\omega}=C^{\infty}(M, \mathbb{R})$ 
and $\{f, g\}$ is the usual Poisson bracket from symplectic geometry. Moreover, the non-degeneracy of $\omega$ also implies that the map

$$
\widehat{\mathfrak{h a m}}(M, \omega) \rightarrow\left(C^{\infty}(M, \mathbb{R}),\{\cdot, \cdot\}\right)
$$

is an isomorphism of Lie algebras.

Besides the classical case $p=0$, such extensions arise in the structure theory of toroidal Lie algebras, where the space

$$
H_{c}^{2}\left(\mathcal{V}(M), \bar{\Omega}^{1}(M, \mathbb{R})\right)
$$

for the $d$-dimensional torus $M=\mathbb{T}^{d}$ plays an important role in the context of classification problems. This space contains in particular the image of the natural map

$$
T_{2}^{M}: H_{\mathrm{dR}}^{3}(M, \mathbb{R}) \rightarrow H_{c}^{2}\left(\mathcal{V}(M), \bar{\Omega}^{1}(M, \mathbb{R})\right)
$$

which in general is not injective (see [1] for a more detailed analysis of this situation for parallelizable manifolds).

Remark 22. If $M$ is compact of dimension $n$ and $\mu$ a volume form on $M$, then the map $\widetilde{f}_{\mu}: \mathcal{V}(M) \rightarrow \Omega^{n-1}(M, \mathbb{R}), X \mapsto i_{X} \mu$ is a linear isomorphism, which leads to linear isomorphisms

$$
\mathfrak{h a m}(M, \mu) \cong B_{\mathrm{dR}}^{n-1}(M, \mathbb{R}) \quad \text { and } \quad \mathfrak{s p}(M, \mu) \cong Z_{\mathrm{dR}}^{n-1}(M, \mathbb{R})
$$

and we obtain a central extension

$$
\mathbf{0} \rightarrow H_{\mathrm{dR}}^{n-2}(M, \mathbb{R}) \hookrightarrow \widehat{\mathfrak{h a m}}(M, \mu) \cong \bar{\Omega}^{n-2}(M, \mathbb{R}) \rightarrow \mathfrak{h a m}(M, \mu) \rightarrow \mathbf{0}
$$

which is the universal central extension of $\mathfrak{h a m}(M, \mu)$ ([7], [13]).

In view of Remark $10 \mathrm{~b}$ ), the following lemma shows that under mild assumptions on $M$, we have

$$
\mathfrak{s p}(M, \omega)=\mathfrak{s p}\left(\mathcal{V}(M), f_{\omega}\right) \quad \text { and } \quad \mathfrak{h a m}(M, \omega)=\mathfrak{h a m}\left(\mathcal{V}(M), f_{\omega}\right) .
$$

Lemma 23. If $M$ is locally smoothly paracompact and $p \in \mathbb{N}_{0}$, then

$$
\Omega^{p+1}(M, E)^{\mathcal{V}(M)}=\{0\} \quad \text { and } \quad \bar{\Omega}^{p}(M, E)^{\mathcal{V}(M)}=H_{\mathrm{dR}}^{p}(M, E) .
$$

Proof: First we show that each closed $p$-form invariant under all vector fields vanishes. Since $M$ is locally smoothly paracompact, standard arguments using smooth bump function reduce the problem to showing that on any open convex 
0-neighborhood $U$ in a locally convex space $V$, each $\mathcal{V}(U)$-invariant $(p+1)$-form $\omega$ vanishes in 0 .

We consider the identity function $Y: U \rightarrow V, x \mapsto x$ as a smooth vector field on $U$. For each constant vector field $X$ on $U$ we then have $[Y, X]=\mathrm{d} X(Y)-$ $\mathrm{d} Y(X)=-X$ and therefore $Y(0)=0$ leads to

$$
0=\left(\mathcal{L}_{Y} \omega\right)\left(X_{1}, \ldots, X_{p+1}\right)(0)=p \omega\left(X_{1}, \ldots, X_{p+1}\right)(0)
$$

This implies that $\omega$ vanishes in 0 .

The second part is proved as Lemma 11, using the exact sequence

$$
\mathbf{0} \rightarrow H_{\mathrm{dR}}^{p}(M, E) \hookrightarrow \bar{\Omega}^{p}(M, E) \stackrel{\overline{\mathrm{d}}}{\longrightarrow} \Omega^{p+1}(M, E)
$$

of $\mathcal{V}(M)$-modules, where $\overline{\mathrm{d}}[\eta]:=\mathrm{d} \eta$ and $\Omega^{p+1}(M, E)^{\mathcal{V}(M)}=\{0\}$.

For the following proposition, we use the concept of smooth singular chains and cycles (cf. [6] or [18]).

Proposition 24. a) For each smooth singular p-cycle $\Sigma$ in $M$, integration over $\Sigma$ defines a continuous linear map

$$
I_{\Sigma}: \bar{\Omega}^{p}(M, E) \rightarrow E, \quad[\beta] \mapsto \int_{\Sigma} \beta
$$

b) If $\omega \in Z_{\mathrm{dR}}^{p+k}(M, E)$, then $\omega_{\Sigma}:=I_{\Sigma} \circ T_{k}^{M}(\omega)$ defines on $\mathfrak{s p}(M, \omega)$ a $p$ cocycle with respect to the trivial module structure on $E$.

c) If, in addition, there exists a smooth singular $(p+1)$-chain $\Gamma$ with $\partial \Gamma=\Sigma$, then $\omega_{\Sigma}$ is a coboundary.

d) If $\omega \in Z_{\mathrm{dR}}^{p+k}(M, E)$, the element $\mathrm{d} \circ T_{k}^{M}(\omega)=(-1)^{k-1} \mathrm{~d}_{\mathcal{V}(M)}\left(\widetilde{T}_{k-1}^{M}(\omega)\right)$ of $Z^{k}\left(\mathcal{V}(M), \Omega^{p+1}(M, E)\right)$ is a coboundary. If the sequence

$$
\mathbf{0} \rightarrow H_{\mathrm{dR}}^{p}(M, E) \hookrightarrow \bar{\Omega}^{p}(M, E) \stackrel{\mathrm{d}}{\longrightarrow} B_{\mathrm{dR}}^{p+1}(M, E) \rightarrow \mathbf{0}
$$

splits topologically and $M$ is finite-dimensional manifold, then the cohomology class $\left[T_{k}^{M}(\omega)\right] \in H_{c}^{k}\left(\mathcal{V}(M), \bar{\Omega}^{p}(M, E)\right)$ comes from an element of $H_{c}^{k}\left(\mathcal{V}(M), H_{\mathrm{dR}}^{p}(M, E)\right)$. This splitting condition is satisfied if $M$ is (smoothly) paracompact and the singular homology group $H_{p}(M)$ is finitely generated. 
Proof: a) is an immediate consequence of Stokes' Theorem.

b) In view of the invariance of $\omega$ and hence of $T_{k}(\omega)$ under $\mathfrak{s p}(M, \omega)$, Lemma 8 implies that $T_{k}(\omega)$ is a cocycle with respect to the trivial action of $\mathfrak{s p}(M, \omega)$ on $\bar{\Omega}^{p}(M, E)$. Composing with $I_{\Sigma}$ therefore leads to a cocycle with values in the trivial module $E$.

c) For $X_{1}, \ldots, X_{k} \in \mathfrak{g}:=\mathfrak{s p}(M, \omega)$, we obtain with Lemmas 8 and 20 the formula

$$
\begin{aligned}
\mathrm{d}\left(i_{X_{k}}\right. & \left.\cdots i_{X_{1}} \omega\right) \\
& =(-1)^{k} \widetilde{T}_{k}^{M}(\mathrm{~d} \omega)\left(X_{1}, \ldots, X_{k}\right)+(-1)^{k-1}\left(\mathrm{~d}_{\mathfrak{g}} \widetilde{T}_{k-1}^{M} \omega\right)\left(X_{1}, \ldots, X_{k}\right) \\
& =(-1)^{k-1}\left(\mathrm{~d}_{\mathfrak{g}} \widetilde{T}_{k-1}^{M} \omega\right)\left(X_{1}, \ldots, X_{k}\right)=(-1)^{k}\left(\mathrm{~d}_{\mathfrak{g}}^{0} \widetilde{T}_{k-1}^{M} \omega\right)\left(X_{1}, \ldots, X_{k}\right) .
\end{aligned}
$$

Since the integration map $I_{\Gamma}$ is equivariant with respect to the trivial $\mathfrak{g}$-module structure on $\bar{\Omega}^{p+1}(M, E)$, we derive with Stokes' Theorem

$$
\begin{aligned}
\omega_{\Sigma}\left(X_{1}, \ldots, X_{k}\right)=\int_{\Sigma} i_{X_{k}} \cdots i_{X_{1}} \omega=\int_{\partial \Gamma} i_{X_{k}} \cdots i_{X_{1}} \omega=\int_{\Gamma} \mathrm{d}\left(i_{X_{k}} \cdots i_{X_{1}} \omega\right) \\
\quad=(-1)^{k} \int_{\Gamma}\left(\mathrm{d}_{\mathfrak{g}}^{0} \widetilde{T}_{k-1}^{M} \omega\right)\left(X_{1}, \ldots, X_{k}\right) \\
\quad=(-1)^{k} \mathrm{~d}_{\mathfrak{g}}^{0}\left(I_{\Gamma} \circ \widetilde{T}_{k-1}^{M} \omega\right)\left(X_{1}, \ldots, X_{k}\right)=(-1)^{k}\left(\mathrm{~d}_{\mathfrak{g}}^{0} \omega_{\Gamma}\right)\left(X_{1}, \ldots, X_{k}\right) .
\end{aligned}
$$

Hence $\omega_{\Sigma}=(-1)^{k} \mathrm{~d}_{\mathfrak{g}}^{0} \omega_{\Gamma}$ is a coboundary.

d) The first part follows as in Proposition 6b) from Lemma 20. If (7) splits topologically, then the exactness of the corresponding long exact cohomology sequence of topological $\mathcal{V}(M)$-modules implies the second assertion.

If $M$ is smooth paracompact and $H_{p}(M)$ is finitely generated, then de Rham's Theorem implies that there exist smooth singular cycles $C_{1}, \ldots, C_{n}$ such that

$$
\bar{\Omega}^{p}(M, E) \rightarrow E^{n} \cong H_{\mathrm{dR}}^{p}(M, E), \quad[\eta] \mapsto\left(\int_{C_{1}} \eta, \ldots, \int_{C_{n}} \eta\right)
$$

yields a topological splitting of (7).

In [14] similar constructions are used to obtain for a compact symplectic manifold $(M, \omega)$ central extensions of $\mathfrak{s p}(M, \omega)$ by cocycles of the form $\omega_{\Sigma}^{k}$, where $\Sigma$ is given by a $2 k$-dimensional orientable submanifold (see also [4]). If $\omega=\mu$ is a volume form on a compact manifold, one similarly obtains the Lichnerowicz cocycles $\Omega_{\Sigma}=I_{\Sigma} \circ \Omega$, where $\Sigma \subseteq M$ is an orientable submanifold of codimension two. 
Corollary 25. For $\omega \in Z_{\mathrm{dR}}^{k}(M, E)$ we obtain for each $m \in M$ a continuous $k$-cocycle

$$
\omega_{m}\left(X_{1}, \ldots, X_{k}\right):=\omega\left(X_{1}, \ldots, X_{k}\right)(m)
$$

of $\mathfrak{s p}(M, \omega)$ with values in the trivial module $E$. If $m$ and $n$ lie in the same connected component of $M$, then $\omega_{m}-\omega_{n}$ is a coboundary.

Proof: We apply the preceding proposition with $\Sigma=[m]-[n]$, viewed as a singular zero-chain, and a path $\Gamma:[0,1] \rightarrow M$ from $m$ to $n$, viewed as a singular one-chain.

Remark 26. Let $N$ be a compact oriented p-dimensional manifold and E a Mackey complete locally convex space. Then we have a natural map

$$
\Phi: \bar{\Omega}^{p}(M, E) \rightarrow C^{\infty}\left(C^{\infty}(N, M), E\right), \quad[\alpha] \mapsto\left(\gamma \mapsto \int_{\gamma} \alpha=\int_{N} \gamma^{*} \alpha\right) .
$$

We further have a homomorphism $\alpha: \mathcal{V}(M) \rightarrow \mathcal{V}\left(C^{\infty}(N, M)\right), \alpha(X)(\phi)=$ $X \circ \phi$ defining on the right hand side a $\mathcal{V}(M)$-module structure for which $\Phi$ is equivariant.

Example 27. Assume $M$ to be connected and write $q_{M}: \widetilde{M} \rightarrow M$ for a simply connected covering. Then the fibers of $q_{M}$ correspond to orbits of the group $\pi_{1}(M)$ of deck transformations and we have a natural isomorphism $\mathcal{V}(\widetilde{M})^{\pi_{1}(M)}$ $\cong \mathcal{V}(M)$, hence in particular an embedding $\iota: \mathcal{V}(M) \hookrightarrow \mathcal{V}(\widetilde{M})$.

Now let $\omega \in Z_{\mathrm{dR}}^{2}(M, E)$ be a closed two-form and $\widetilde{\omega}:=q_{M}^{*} \omega$ its pull-back to $\widetilde{M}$. Since $H_{\mathrm{dR}}^{1}(\widetilde{M}, E)=\{0\}$, we have $\mathfrak{s p}(\widetilde{M}, \widetilde{\omega})=\mathfrak{h a m}(\widetilde{M}, \widetilde{\omega})$. In particular, we can use the embedding

$$
\mathfrak{s p}(M, \omega) \cong \mathfrak{s p}(\widetilde{M}, \widetilde{\omega})^{\pi_{1}(M)} \hookrightarrow \mathfrak{h} \mathfrak{a m}(\widetilde{M}, \widetilde{\omega})
$$

to pull back the central extension $\widehat{\mathfrak{h a m}}(\widetilde{M}, \widetilde{\omega})$ to obtain a central extension of $\mathfrak{s p}(M, \omega)$ by $H_{\mathrm{dR}}^{0}(\widetilde{M}, E) \cong E$. This extension is given by

$$
\widetilde{\mathfrak{s p}}(M, \omega)=\left\{(f, X) \in C^{\infty}(\widetilde{M}, E) \times \mathfrak{s p}(M, \omega) ; \mathrm{d} f=i_{\widetilde{X}} \widetilde{\omega}=q_{M}^{*}\left(i_{X} \omega\right)\right\} .
$$

To obtain a cocycle for this extensions, we pick a point $m_{0} \in M$, a point $\widetilde{m}_{0} \in \widetilde{M}$ with $q_{M}\left(\widetilde{m}_{0}\right)=m_{0}$, and define for $X \in \mathfrak{s p}(M, \omega)$

$$
f_{X}: \widetilde{M} \rightarrow E, \quad \widetilde{m} \mapsto \int_{\tilde{m}_{0}}^{\tilde{m}} q_{M}^{*}\left(i_{X} \omega\right)=\int_{\widetilde{m}_{0}}^{\widetilde{m}} i_{\widetilde{X}} \widetilde{\omega} .
$$


Then $\sigma: \mathfrak{s p}(M, \omega) \rightarrow \widetilde{\mathfrak{s p}}(M, \omega), X \mapsto\left(f_{X}, X\right)$ is a continuous linear section, and the corresponding cocycle is

$$
\omega_{\sigma}(X, Y):=[\sigma(X), \sigma(Y)]-\sigma([X, Y])=\left(\widetilde{\omega}(\widetilde{Y}, \widetilde{X})-f_{[X, Y]}, 0\right)
$$

where the function $\widetilde{\omega}(\widetilde{X}, \widetilde{Y})-f_{[X, Y]}$ is constant because $\mathrm{d} \widetilde{\omega}(\widetilde{Y}, \widetilde{X})=i_{[\widetilde{X}, \widetilde{Y}]} \widetilde{\omega}$. This shows that

$$
\omega_{\sigma}(X, Y)=\widetilde{\omega}(\widetilde{Y}, \widetilde{X})\left(\widetilde{m}_{0}\right)=\omega(Y, X)\left(m_{0}\right) .
$$

Hence $\left[\omega_{\sigma}\right]=-\left[\omega_{m}\right]$ for each $m \in M$ (cf. Corollary 25).

If $C_{*}^{\infty}(M, E)$ denotes the subspace of those functions vanishing in $m_{0}$, then we clearly have $C^{\infty}(M, E) \cong C_{*}^{\infty}(M, E) \oplus E$ as trivial $\mathfrak{s p}(M, \omega)$-modules, where the projection onto the second summand is evaluation in $m$. The two-cocycle $\omega_{0}$ decomposes accordingly as $\omega_{0}=\left(\omega_{*}, \omega_{m}\right)$. We know from Proposition $\left.24 \mathrm{~d}\right)$ that $\omega_{*}$ is trivial whenever $H_{1}(M)$ is finitely generated. Considering the embed$\operatorname{ding} q_{M}^{*}: C^{\infty}(M, E) \hookrightarrow C^{\infty}(\widetilde{M}, E)$, we easily obtain an explicit coboundary because

$$
\eta: \mathfrak{s p}(M, \omega) \rightarrow C_{*}^{\infty}(\widetilde{M}, E), \quad \eta(X)(\widetilde{m}):=\int_{\widetilde{m}_{0}}^{\widetilde{m}} q_{M}^{*}\left(i_{X} \omega\right)
$$

satisfies

$$
\eta([Y, X])(\widetilde{m})=f_{[Y, X]}(\widetilde{m})=\widetilde{\omega}(\widetilde{X}, \widetilde{Y})(\widetilde{m})-\widetilde{\omega}(\widetilde{X}, \widetilde{Y})\left(\widetilde{m}_{0}\right)=\omega_{*}(X, Y)(\widetilde{m}) .
$$

Example 28. Let $Z$ be an abelian Lie group with Lie algebra $\mathfrak{z}$ and $q: P \rightarrow$ $M$ a principal $Z$-bundle with principal connection one-form $\theta \in \Omega^{1}(P, \mathfrak{z})$ and curvature form $\omega \in \Omega^{2}(M, \mathfrak{z})$, i.e., $q^{*} \omega=-\mathrm{d} \theta$. Let $\mathfrak{a} \mathfrak{u}(P):=\mathcal{V}(P)^{Z}$ denote the Lie algebra of $Z$-invariant vector fields on $P$. Then there exists for each $X \in \mathfrak{a u t}(P)$ a unique vector field $q_{*}(X) \in \mathcal{V}(M)$ which is q-related to $X$. This leads to the exact sequence of topological Lie algebras

$$
\mathbf{0} \rightarrow C^{\infty}(M, \mathfrak{z}) \hookrightarrow \mathcal{V}(P)^{Z} \stackrel{q_{*}}{\longrightarrow} \mathcal{V}(M) \rightarrow \mathbf{0}
$$

where we identify the space $\operatorname{ker} q_{*}$ of vertical Z-invariant vector fields with the abelian Lie algebra $C^{\infty}(M, \mathfrak{z})$.

We obtain a continuous linear section $\sigma: \mathcal{V}(M) \rightarrow \mathcal{V}(P)^{Z}$ by assigning to $X \in$ $\mathcal{V}(M)$ the unique vector field $\widetilde{X} \in \mathcal{V}(P)^{Z}$ with $\theta(\widetilde{X})=0$ and $q_{*} \widetilde{X}=X(a$ horizontal vector field). Then

$$
\begin{aligned}
\omega(X, Y) & =\left(q^{*} \omega\right)(\sigma(X), \sigma(Y))=\theta([\sigma(X), \sigma(Y)]) \\
& =\theta([\sigma(X), \sigma(Y)]-\sigma([X, Y]))
\end{aligned}
$$


is the two-cocycle of the abelian extension (8), so that

$$
\mathcal{V}(P)^{Z} \cong C^{\infty}(M, \mathfrak{z}) \oplus_{\omega} \mathcal{V}(M)
$$

(cf. [10], Section VI for a similar discussion of non-abelian structure groups). In view of Remark 14 , the subalgebra $\mathfrak{h a m}(M, \omega)$ consists of those vector fields $X \in \mathcal{V}(M)$ which have a lift $\widehat{X} \in \mathcal{V}(P)^{Z}$ preserving the connection one-form $\theta$, i.e., the splitting defined by $\sigma$. This leads to the central extension

$$
\mathbf{0} \rightarrow H_{\mathrm{dR}}^{0}(M, \mathfrak{z}) \hookrightarrow \widehat{\mathfrak{h a m}}(M, \omega) \rightarrow \mathfrak{h a m}(M, \omega) \rightarrow \mathbf{0}
$$

describing the Lie subalgebra $\widehat{\mathfrak{h a m}}(M, \omega) \subseteq \mathcal{V}(P)^{Z}$ of infinitesimal automorphisms of the pre-quantum data $(P, \theta)$.

\section{Actions of Lie Algebras on Manifolds}

In this short section we briefly discuss a more general situation, where $\mathfrak{g}$ is a locally convex Lie algebra and we are given an infinitesimal action of $\mathfrak{g}$ on the manifold $M$, i.e., a continuous homomorphism of Lie algebras $\alpha: \mathfrak{g} \rightarrow \mathcal{V}(M)$.

\subsection{Equivariant Differential Forms}

Further let $E$ be a topological $\mathfrak{g}$-module w.r.t. $(x, v) \mapsto \rho_{E}(x) . v$. We thus obtain on $\Omega^{p}(M, E)$ a $\mathfrak{g}$-module structure by

$$
x . \omega:=\mathcal{L}_{\alpha(x)} \omega+\rho_{E}(x) \circ \omega .
$$

In particular $V:=C^{\infty}(M, E)=\Omega^{0}(M, E)$ is a $\mathfrak{g}$-module. Let $\mathrm{d}_{\mathfrak{g}}^{1}$ denote the differential on $C_{c}^{p}(\mathfrak{g}, V)$ corresponding to the trivial action of $\mathfrak{g}$ on $E$ and $\mathrm{d}_{\mathfrak{g}}^{0}$ for the differential corresponding to the trivial action of $\mathfrak{g}$ on $V$. For the corresponding action on $V$ we accordingly write $\rho_{V}(x) f=\rho_{V}^{0}(x) f+\rho_{E}(x) \circ f$ and $\rho_{V}^{E}(x) f=$ $\rho_{E}(x) \circ f$. Then $\mathrm{d}_{\mathfrak{g}}=\mathrm{d}_{\mathfrak{g}}^{0}+\rho_{V} \wedge$ where $\rho_{V} \wedge: C_{c}^{p}(\mathfrak{g}, V) \rightarrow C_{c}^{p+1}(\mathfrak{g}, V)$ denotes the map induced by the bilinear map $\mathfrak{g} \times V \rightarrow V,(x, v) \mapsto \rho_{V}(x) v$ (cf. [10], Exercise I.3).

With respect to the pull back maps $\alpha^{*}: \Omega^{p}(M, E) \rightarrow C_{c}^{p}(\mathfrak{g}, V)$ we have

$$
\mathrm{d}_{\mathfrak{g}}\left(\alpha^{*} \omega\right)=\alpha^{*}(\mathrm{~d} \omega)+\rho_{E} \wedge \alpha^{*} \omega=\mathrm{d}_{\mathfrak{g}}^{1}\left(\alpha^{*} \omega\right)+\rho_{E} \wedge \alpha^{*} \omega .
$$

We are mostly interested in the subspace $\Omega^{p}(M, E)^{\mathfrak{g}}$ of $\mathfrak{g}$-invariant forms which pull back to invariant Lie algebra cochains in $C_{c}^{p}(\mathfrak{g}, V)^{\mathfrak{g}}$. In view of Lemma 8 , we obtain for each invariant form $\omega$

$$
\mathrm{d}_{\mathfrak{g}}^{1}\left(\alpha^{*} \omega\right)+\rho_{E} \wedge \alpha^{*} \omega=\mathrm{d}_{\mathfrak{g}}\left(\alpha^{*} \omega\right)=-\mathrm{d}_{\mathfrak{g}}^{0}\left(\alpha^{*} \omega\right)
$$


which leads to

$$
\alpha^{*}(\mathrm{~d} \omega)=\mathrm{d}_{\mathfrak{g}}^{1}\left(\alpha^{*} \omega\right)=-\left(\mathrm{d}_{\mathfrak{g}}^{0}+\rho_{E} \wedge\right)\left(\alpha^{*} \omega\right)=-\mathrm{d}_{\mathfrak{g}}^{\rho_{V}^{E}}\left(\alpha^{*} \omega\right)
$$

describing how the exterior differential on $\Omega^{p}(M, E)$ intertwines $\alpha^{*}$.

For any point $m \in M$ we have an evaluation map ev $\mathrm{ev}_{m}: \Omega^{p}(M, E) \rightarrow C_{c}^{p}(\mathfrak{g}, E)$

$$
\mathrm{ev}_{m}(\omega)\left(x_{1}, \ldots, x_{p}\right):=\omega\left(\alpha\left(x_{1}\right), \ldots, \alpha\left(x_{p}\right)\right)(m)=\left(\left(\alpha^{*} \omega\right)\left(x_{1}, \ldots, x_{p}\right)\right)(m) .
$$

Since the evaluation map $\mathrm{ev}_{m}: V \rightarrow E, f \mapsto f(m)$ is $\mathfrak{g}$-equivariant with respect to $\rho_{V}^{E}$, (9) implies that

$$
\begin{aligned}
\mathrm{d}_{\mathfrak{g}}\left(\operatorname{ev}_{m}(\omega)\right)=\mathrm{d}_{\mathfrak{g}}\left(\operatorname{ev}_{m} \circ \alpha^{*} \omega\right) & =\operatorname{ev}_{m} \circ \mathrm{d}_{\mathfrak{g}}^{\rho_{V}^{E}}\left(\alpha^{*} \omega\right) \\
& =-\mathrm{ev}_{m} \circ \alpha^{*}(\mathrm{~d} \omega)=-\mathrm{ev}_{m}(\mathrm{~d} \omega)
\end{aligned}
$$

showing that $\mathrm{ev}_{m}$ induces maps

$$
H_{\mathrm{dR}}^{p}(M, E)^{\mathfrak{g}} \rightarrow H_{c}^{p}(\mathfrak{g}, E), \quad[\omega] \mapsto\left[\mathrm{ev}_{m}(\omega)\right] .
$$

Such evaluation maps are used in [15] to realize abelian extensions of Lie group geometrically.

Example 29. Of particular interest is the special case where $M=G$ is a Lie group with identity e and $\mathfrak{g}=\mathbf{L}(G) \cong T_{e}(G)$ is the Lie algebra of $G$, which we identify with the space of left invariant vector fields on $G$. Writing $x_{r}$ for the right invariant vector field with $x_{r}(e)=x$, the map $\sigma: \mathfrak{g} \rightarrow \mathcal{V}(G), x \mapsto-x_{r}$ is a continuous homomorphism of Lie algebras and $\Omega^{p}(G, E)^{\mathfrak{g}}$ is the space of left equivariant differential forms, characterized by

$$
\mathcal{L}_{x_{r}} \omega=\rho_{E}(x) \circ \omega \quad \text { for } \quad x \in \mathfrak{g} .
$$

This space has the remarkable property that evaluation in e leads to an isomorphism

$$
\left(\Omega(G, E)^{\mathfrak{g}}, \mathrm{d}\right) \rightarrow\left(C_{c}(\mathfrak{g}, E), \mathrm{d}_{\mathfrak{g}}\right)
$$

of chain complexes (cf. [2], [9]).

\subsection{Generalized Momentum Maps}

Definition 30. Assume that $E$ is a trivial $\mathfrak{g}$-module and $\omega$ is a closed E-valued $(p+2)$-form. Let $\alpha: \mathfrak{g} \rightarrow \mathfrak{h a m}(M, \omega)$ be a continuous homomorphism. A continuous linear map $\mu: \mathfrak{g} \rightarrow \bar{\Omega}^{p}(M, E)$ is said to be a generalized momentum map for $\alpha$ if 
1) $\mathrm{d} \mu(x)=i_{\alpha(x)} \omega$ for $x \in \mathfrak{g}$.

2) $\mu([x, y])=\{\mu(x), \mu(y)\}=\Omega(\alpha(y), \alpha(x))$, i.e., $\mu$ is a homomorphism of Lie algebras with respect to the Poisson bracket on $\bar{\Omega}^{p}(M, E)_{\omega}$.

We may pull back the central extension $\widehat{\mathfrak{h a m}}(M, \omega)$ of $\mathfrak{h a m}(M, \omega)$ by $H_{\mathrm{dR}}^{p}(M, E)$ defined by $\omega$ to a central extension of $\mathfrak{g}$. From the definition of this central extension it immediately follows that $\mu: \mathfrak{g} \rightarrow \bar{\Omega}^{p}(M, E)$ is a generalized momentum map for $\alpha$ if and only if $(\mu, \alpha): \mathfrak{g} \rightarrow \widehat{\mathcal{V}}(M)$ has values in $\widehat{\mathfrak{h a m}}(M, \omega)$ and is a homomorphism of Lie algebras. Therefore the existence of a momentum map is equivalent to the triviality of the central extension $\alpha^{*} \widehat{\mathfrak{h a m}}(\mathfrak{g}, \omega)$ of $\mathfrak{g}$. Note also that 2) means that the two-cocycle $\alpha^{*} \Omega \in Z_{c}^{2}\left(\mathfrak{g}, \bar{\Omega}^{p}(M, E)\right)$ satisfies $\alpha^{*} \Omega=\mathrm{d}_{\mathfrak{g}} \mu$. Here $\alpha(\mathfrak{g}) \subseteq \mathfrak{h a m}(M, \omega)$ already implies that $\alpha^{*} \Omega$ can be reduced to a cocycle with values in the trivial module $H_{\mathrm{dR}}^{p}(M, E)$, and the triviality of this cocycle is equivalent to the existence of a momentum map.

If $\omega=\mathrm{d} \theta$ is an exact form and $\theta$ is also $\mathfrak{g}$-invariant, then $\mu(x):=\left[-i_{\alpha(x)} \theta\right]$ satisfies

$$
\mathrm{d} \mu(x)=-\mathrm{d} i_{\alpha(x)} \theta=-\mathcal{L}_{\alpha(x)} \theta+i_{\alpha(x)} \mathrm{d} \theta=i_{\alpha(x)} \omega
$$

and $0=\mathcal{L}_{\alpha(y)} \theta=\mathrm{d} i_{\alpha(y)} \theta+i_{\alpha(y)} \mathrm{d} \theta=\mathrm{d} i_{\alpha(y)} \theta+i_{\alpha(y)} \omega$ further yields

$$
\begin{aligned}
\mu([x, y]) & =-\left[i_{\alpha([x, y])} \theta\right]=-\left[\left[\mathcal{L}_{\alpha(x)}, i_{\alpha(y)}\right] \theta\right]=-\left[\mathcal{L}_{\alpha(x)} i_{\alpha(y)} \theta\right] \\
& =-\left[i_{\alpha(x)} \mathrm{d} i_{\alpha(y)} \theta\right]=\left[i_{\alpha(x)} i_{\alpha(y)} \omega\right]=\Omega(\alpha(y), \alpha(x))
\end{aligned}
$$

so that $\mu$ is a momentum map.

Example 31. If $M$ is a Banach manifold, then its cotangent bundle $T^{*}(M)$ carries the Liouville one-form $\theta_{M}$, which is invariant under the hamiltonian lift $\alpha$ : $\mathcal{V}(M) \rightarrow \mathcal{V}\left(T^{*}(M)\right)$. This leads to a moment map

$$
\mu: \mathcal{V}(M) \rightarrow C^{\infty}\left(T^{*}(M), \mathbb{R}\right), \quad X \mapsto-\theta_{M}(\alpha(X)) .
$$

A similar situation arises for the infinitesimal action $\widehat{\mathfrak{h a m}}(M, \omega) \rightarrow \mathcal{V}(P)$ of a pre-quantum bundle $(P, \theta)$ which preserves the connection one-form $\theta$ (cf. Example 28).

Remark 32. Via $\alpha$ we obtain on $\bar{\Omega}^{p}(M, E)$ a natural structure of a $\mathfrak{g}$-module by

$$
x .[\eta]=\left[\mathcal{L}_{\alpha(x)} \eta\right]=\left[i_{\alpha(x)} \mathrm{d} \eta\right] .
$$

Therefore $\mathrm{d} \mu(y)=i_{\alpha(y)} \omega$ implies that

$$
x . \mu(y)=\left[i_{\alpha(x)} i_{\alpha(y)} \omega\right]=\Omega(y, x)=\{\mu(x), \mu(y)\} .
$$

We conclude that if $\mu$ satisfies 1 ), then its equivariance is equivalent to 2 ). 
Remark 33. Assume that $p=0$ and that $M$ is connected. Then $\bar{\Omega}^{p}(M, E)=$ $C^{\infty}(M, E)$ and $\mu$ corresponds to a map

$$
\widehat{\mu}: M \rightarrow C_{c}^{1}(\mathfrak{g}, E), \quad \widehat{\mu}(m)(x):=\mu(x)(m)
$$

being usually called the momentum map. The classical setting is obtained for $E=\mathbb{R}$ and $(M, \omega)$ symplectic. Each $x \in \mathfrak{g}$ defines a linear vector field $\operatorname{ad}_{E}^{*}(x)$ on $C_{c}^{1}(\mathfrak{g}, E)=\operatorname{Lin}(\mathfrak{g}, E)$ by $\operatorname{ad}_{E}^{*}(x)(f):=f \circ \operatorname{ad} x$, which leads to a homomorphism $\mathfrak{g} \rightarrow \mathcal{V}\left(C_{c}^{1}(\mathfrak{g}, E)\right)$. From this viewpoint we have

$$
\operatorname{ad}_{E}^{*}(x)(\widehat{\mu}(m))(y)=\widehat{\mu}(m)([x, y])=\mu([x, y])(m)
$$

and

$T_{m}(\mu)\left(\alpha(x)_{m}\right)(y)=\mathrm{d} \mu(y)(\alpha(x))(m)=\omega(\alpha(y), \alpha(x))(m)=\{\alpha(x), \alpha(y)\}(m)$.

Hence we also see in this context that $\mu$ is equivariant, i.e., the vector fields $\alpha(x)$ and $\operatorname{ad}_{E}^{*}(x)$ are $\mu$-related, if and only if 2 ) is satisfied.

\section{Appendix. The Topology on Vector Fields and Differential Forms}

If $X$ and $Y$ are topological spaces, then the compact open topology on the space $C(X, Y)$ is defined as the topology generated by the sets of the form

$$
W(K, U):=\{f \in C(X, Y) ; f(K) \subseteq U\}
$$

where $K$ is a compact subset of $X$ and $U$ an open subset of $Y$. We write $C(X, Y)_{c}$ for the topological space obtained by endowing $C(X, Y)$ with the compact open topology.

If $M$ and $N$ are smooth manifolds, then every smooth map $f: M \rightarrow N$ defines a series of smooth maps $T^{k} f: T^{k} M \rightarrow T^{k} N$ on the iterated tangent bundles. We thus obtain an embedding

$$
C^{\infty}(M, N) \hookrightarrow \prod_{k \in \mathbb{N}_{0}} C\left(T^{k} M, T^{k} N\right)_{c}
$$

that we use to define a topology on $C^{\infty}(M, N)$.

Since every compact subset of $M$ is contained in a finite union of chart domains, the topology is generated by sets of the form $W(K, U)$ in $C\left(T^{k} M, T^{k} N\right)$, where $K$ lies in a chart domain.

If $E$ is a locally convex space, the topology on $C^{\infty}(M, E)$ coincides with the topology of uniform convergence of all partial derivatives on each compact subset 
of a chart domain. It follows in particular that $C^{\infty}(M, E)$ is a locally convex space.

Since smooth vector fields are smooth functions $X: M \rightarrow T M$ (sections of the tangent bundle), we have a natural embedding $\mathcal{V}(M) \hookrightarrow C^{\infty}(M, T M)$, defining a topology on $\mathcal{V}(M)$. If $U \subseteq M$ is a chart domain diffeomorphic to an open subset of the locally convex space $E$, then $T U \cong U \times E$, and smooth vector fields on $U$ correspond to smooth functions $U \rightarrow E$. This shows that, endowed with its natural topology, $\mathcal{V}(M)$ is a locally convex space. Since on $U$ the Lie bracket of two vector fields is given by the formula

$$
[X, Y](p):=\mathrm{d} Y(p) X(p)-\mathrm{d} X(p) Y(p)
$$

$\mathcal{V}(M)$ is a topological Lie algebra if $M$ is finite-dimensional, and if $M$ is infinitedimensional, the bracket is in general not continuous.

If $E$ is a locally convex space and $\Omega^{p}(M, E)$ the space of smooth $E$-valued $p$ forms, then we identify for each chart domain $U$ the set $\Omega^{p}(U, E)$ with a subspace of $C^{\infty}\left(U \times E^{p}, E\right)$, which leads to a locally convex topology on $\Omega^{p}(M, E)$ by embedding it into the product of all sets $\Omega^{p}(U, E)$, where $U$ is a chart domain. The formula for the Lie derivative shows that if $M$ is finite-dimensional, this turns $\Omega^{p}(M, E)$ into a topological $\mathcal{V}(M)$-module.

For more details on the subject of this appendix, we refer to the forthcoming book [3].

\section{Acknowledgements}

We thank the organizers of the $24^{\text {th }}$ Workshop on Geometric Methods in Physics in Bialowieza for organizing this interesting event in such a pleasant environment.

\section{References}

[1] Billig Y. and K.-H. Neeb, On the Cohomology of Vector Fields on Tori, in preparation.

[2] Chevalley C. and Eilenberg S., Cohomology Theory of Lie Groups and Lie Algebras, Transactions of the Amer. Math. Soc. 63 (1948) 85-124.

[3] Glöckner H. and Neeb, K.-H. Infinite-dimensional Lie Groups, book in preparation.

[4] Haller S. and Vizman C., Nonlinear Grassmannians as Coadjoint Orbits, Math. Annalen 329 (2004) 771-785. 
[5] Hochschild G. and Serre J.-P., Cohomology of Lie algebras, Ann. Math. 57 (1953) 591-603.

[6] Kriegl A. and Michor P., The Convenient Setting of Global Analysis, Math. Surveys and Monographs 53, Amer. Math. Soc., 1997.

[7] Lichnerowicz A., Algèbre de Lie des automorphismes infinitésimaux d'une structure unimodulaire, Ann. Inst. Fourier 24 (1974) 219-266.

[8] Neeb K.-H., Central Extensions of Infinite-Dimensional Lie Groups, Ann. Inst. Fourier 52 (2002) 1365-1442.

[9] Neeb K.-H., Abelian Extensions of Infinite-Dimensional Lie Groups, Travaux mathématiques 15 (2004) 69-194.

[10] Neeb K.-H., Extensions of Topological Lie Algebras, Commun. in Alg., to appear.

[11] Neeb K.-H., Non-Abelian Extensions of Infinite-Dimensional Lie Groups, submitted.

[12] Neeb K.-H. and Vizman C., Flux Homomorphisms and Principal Bundles over Infinite-Dimensional Manifolds, Monatshefte für Math. 139 (2003) 309-333.

[13] Roger C., Extensions centrales d'algèbres et de groupes de Lie de dimension infinie, algèbres de Virasoro et généralisations, Reports on Math. Phys. 35 (1995), 225-266.

[14] Vizman C., Central Extensions of Some Diffeomorphism Groups, Preprint.

[15] Vizman C., A Geometric Construction of Abelian Extensions of Lie Groups, Preprint.

[16] Vizman C., Central Extension of the Lie Algebra of Symplectic Vector Fields, J. Lie Theory 16 (2006) 297-309.

[17] Wagemann F., On Lie Algebra Crossed Modules, Comm. in Algebra, to appear.

[18] Warner F., Foundations of Differentiable Manifolds and Lie Groups, Springer, Berlin, 1983.

Karl-Hermann Neeb

Fachbereich Mathematik

Technische Universität Darmstadt

Schlossgartenstrasse 7

64285 Darmstadt

GERMANY

E-mail address: neeb@mathematik.tu-darmstadt.de 